\title{
MusMorph, a database of standardized mouse morphology data for morphometric meta-analyses
}

Jay Devine ${ }^{1,2,3}$, Marta Vidal-García ${ }^{1,2,3}$, Wei Liu ${ }^{1,2,3}$, Amanda Neves ${ }^{4}$, Lucas D. Lo Vercio ${ }^{1,2,3}$, Rebecca M. Green ${ }^{5}$, Heather A. Richbourg ${ }^{6}$, Marta Marchini ${ }^{1,2,3}$, Colton M. Unger ${ }^{2,7}$, Audrey C. Nickle $^{8,9}$, Bethany Radford ${ }^{1,10}$, Nathan M. Young ${ }^{6}$, Paula N. Gonzalez ${ }^{11}$, Robert E. Schuler ${ }^{12}$, Alejandro Bugacov ${ }^{12}$, Campbell Rolian ${ }^{2,13}$, Christopher J. Percival ${ }^{14}$, Trevor Williams ${ }^{15}$, Lee Niswander ${ }^{16}$, Anne L. Calof ${ }^{17,18}$, Arthur D. Lander ${ }^{17,34}$, Axel Visel ${ }^{19,20,33}$, Frank R. Jirik ${ }^{1,2,10}$, 9 James M. Cheverud ${ }^{22}$, Ophir Klein ${ }^{23,24}$, Ramon Y. Birnbaum ${ }^{25}$, Amy E. Merrill ${ }^{8,9}$, Rebecca R. Ackermann $^{26,27}$, Daniel Graf ${ }^{28,29}$, Myriam Hemberger ${ }^{1,10}$, Wendy Dean ${ }^{1,10}$, Nils D. Forkert ${ }^{1,30}$, Stephen A. Murray ${ }^{31}$, Henrik Westerberg ${ }^{32}$, Ralph S. Marcucio ${ }^{6}$, Benedikt Hallgrímsson ${ }^{1,2,3}$

1. Alberta Children's Hospital Research Institute, University of Calgary, Calgary, AB, CANADA

2. The McCaig Institute for Bone and Joint Health, University of Calgary, Calgary, AB, CANADA

3. Department of Cell Biology and Anatomy, Cumming School of Medicine, University of Calgary, Calgary, AB, CANADA

4. Department of Biology, McMaster University, Hamilton, ON, CANADA

5. School of Dental Medicine, University of Pittsburgh, Pittsburgh, PA, USA

6. Department of Orthopedics, University of California, San Francisco, San Francisco, CA, USA

7. Department of Biological Sciences, University of Calgary, Calgary, AB, CANADA

8. Center for Craniofacial Molecular Biology, Herman Ostrow School of Dentistry, University of Southern California, Los Angeles, CA, USA

9. Department of Biochemistry and Molecular Medicine, Keck School of Medicine, University of Southern California, Los Angeles, CA, USA

10. Department of Biochemistry and Molecular Biology, Cumming School of Medicine, University of Calgary, Calgary, AB, CANADA

11. Institute for Studies in Neuroscience and Complex Systems (ENyS) CONICET, Buenos Aires, ARGENTINA

12. Information Sciences Institute, Viterbi School of Engineering, University of Southern California, Marina del Rey, CA, USA

13. Department of Comparative Biology and Experimental Medicine, Faculty of Veterinary Medicine, University of Calgary, Calgary, AB, CANADA

14. Department of Anthropology, Stony Brook University, New York, NY, USA

15. Department of Craniofacial Biology, University of Colorado, Aurora, CO, USA

16. Department of Molecular, Cellular and Developmental Biology, University of Colorado Boulder, Boulder, CO, USA

17. Department of Anatomy and Neurobiology, University of California, Irvine, Irvine, CA, USA

18. Center for Complex Biological Systems, University of California, Irvine, Irvine, CA, USA

19. Environmental Genomics and Systems Biology Division, Lawrence Berkeley National Laboratory, Berkeley, CA, USA

20. U.S. Department of Energy Joint Genome Institute, Lawrence Berkeley National Laboratory, Berkeley, CA, USA

21. Department of Biochemistry and Molecular Biology, Cumming School of Medicine, University of Calgary, Calgary, AB, CANADA

22. Department of Biology, Loyola University Chicago, Chicago, IL, USA

23. Program in Craniofacial Biology and Department of Orofacial Sciences, University of California, San Francisco, San Francisco, CA, USA

24. Department of Pediatrics and Institute for Human Genetics, University of California, San Francisco, San Francisco, CA, USA

25. Department of Life Sciences, Faculty of Natural Sciences, The Ben-Gurion University of the Negev, Beer-Sheva, ISRAEL

26. Department of Archaeology, University of Cape Town, Cape Town, Rondebosch, SOUTH AFRICA

27. Human Evolution Research Institute, University of Cape Town, Cape Town, Rondebosch, SOUTH AFRICA

28. School of Dentistry, Faculty of Medicine and Dentistry, University of Alberta, Edmonton, AB, CANADA

29. Department of Medical Genetics, Faculty of Medicine and Dentistry, University of Alberta, Edmonton, AB, CANADA

30. Department of Radiology, Cumming School of Medicine, University of Calgary, Calgary, AB, CANADA

31. The Jackson Laboratory, Bar Harbor, ME, USA

32. Department of Bioimaging Informatics, MRC Harwell Institute, Oxfordshire, UK

33. School of Natural Sciences, University of California, Merced, Merced, CA, USA

34. Department of Developmental and Cell Biology, University of California, Irvine, CA, USA

Corresponding author: Benedikt Hallgrímsson (bhallgri@ ucalgary.ca) 


\section{Abstract}

56 Complex morphological traits are the product of many genes with transient or lasting developmental effects

57 that interact in anatomical context. Mouse models are a key resource for disentangling such effects, because

58 they offer myriad tools for manipulating the genome in a controlled environment. Unfortunately,

59 phenotypic data are often obtained using laboratory-specific protocols, resulting in self-contained datasets

60 that are difficult to relate to one another for larger scale analyses. To enable meta-analyses of morphological

61 variation, particularly in the craniofacial complex and brain, we created MusMorph, a database of

62 standardized mouse morphology data spanning numerous genotypes and developmental stages, including

63 E10.5, E11.5, E14.5, E15.5, E18.5, and adulthood. To standardize data collection, we implemented an atlas-

64 based phenotyping pipeline that combines techniques from image registration, deep learning, and

65 morphometrics. Alongside stage-specific atlases, we provide aligned micro-computed tomography images,

66 dense anatomical landmarks, and segmentations (if available) for each specimen $(N=10,056)$. Our workflow

67 is open-source to encourage transparency and reproducible data collection. The MusMorph data and scripts

68 are available on FaceBase (www.facebase.org, doi.org/10.25550/3-HXMC) and GitHub

69 (https://github.com/jaydevine/MusMorph).

70 Keywords: Mouse, phenomics, craniofacial, imaging pipelines, deep learning, morphometrics, 71 micro-computed tomography, FaceBase 


\section{$81 \quad$ Background \& Summary}

82 Understanding how genes, development, and the environment produce variation in complex morphological traits is a core challenge in biology with evolutionary and clinical implications. Explanations for the generation of variation tend to cohere around the genotype-phenotype map concept. Genetic variation and genetic effects, like epistasis and pleiotropy, drive variation in developmental processes that act at different times and scales in anatomical context ${ }^{1-3}$. Specific developmental and genetic mechanisms then operate alongside embedded mechanisms, such as nonlinearities ${ }^{4,5}$ and gene redundancy ${ }^{6}$, to modulate these effects to express a phenotype ${ }^{7-9}$. Despite recent insights into these phenomena, the developmental-genetic basis for morphological variation remains largely unknown, as there are likely many overlapping and coordinated mechanisms involved, each with relative contributions ${ }^{10}$. To help disentangle these mechanisms, it is

91 important to build and integrate large phenotypic databases for model organisms ${ }^{11-14}$. In this work, we present MusMorph, a database of standardized mouse morphology data for meta-analyses of morphological variability and variation, particularly in the craniofacial complex and brain. variation because of its $99 \%$ genetic homology with humans, short gestation, and rich set of tools for manipulating the genome in a controlled environment. Unfortunately, phenotypic data are often biased by laboratory-specific data collection protocols. The International Mouse Phenotyping Consortium (IMPC, www.mousephenotype.org) was born out of a need to determine the relationship between genotype and phenotype with standardized phenotypic data. Using micro-computed tomography ( $\mu \mathrm{CT})$ and optical projection tomography, the consortium has studied the anatomy of mouse lines heterozygous or

101 homozygous for a single gene mutation, particularly at embryonic day E9.5, E14.5-15.5, and E18.5

102 Less emphasis has been placed on $\mu \mathrm{CT}$ imaging and analysis of adults and mid-gestation (E10 to E11)

103 mutants, where critical developmental events, like fusion of the craniofacial prominences, occur. Mouse 104 lines with normal (non-pathological) levels of variation, such as recombinant inbred strains and outbred 
105

106

strains with high heterozygosity ${ }^{21-23}$, have also been poorly characterized. Quantifying such variation is important, because it drives disease susceptibility and course of disease in humans.

Recently, model organism phenotyping has transitioned from manual linear measurements to fully automated computational pipelines. One common approach is voxel-based morphometry ${ }^{24,25}$. Voxel-based morphometry is based on the analysis of deformation fields obtained via image registration. After spatially aligning images to an average atlas, the deformation fields can be quantitatively compared between groups on a voxel-wise basis to identify differences in morphology. Voxel-based morphometry remains a pillar of shape analysis, because it can localize small regions of shape change without any a priori knowledge of the anatomy, but it is prone to the multiple testing problem ${ }^{26,27}$. Another approach is atlas-based geometric morphometrics, which instead uses registration fields to automatically derive landmarks, or Cartesian coordinate points that are homologous across samples. Geometric morphometrics is central to evolutionary biology and developmental biology, among other fields, because landmarks allow for statistically tractable quantifications of morphological variation, as well as intuitive visualizations ${ }^{28}$. These advantages continue to fuel development of novel geometric morphometric pipelines and extensions ${ }^{29-33}$. Yet large-scale morphometric analyses remain rare due to the sparsity of standardized landmark data.

Here, we introduce MusMorph, a database of standardized mouse morphology data generated with an open-source, atlas-based phenotyping pipeline that integrates techniques from image registration, deep learning, and morphometrics. We compiled the database $(N=10,056)$ using $\mu \mathrm{CT}$ scans of mice from a variety of strain/genotype combinations and developmental stages, including E10.5, E11.5, E14.5, E15.5, E18.5, and adulthood. Most of MusMorph is composed of head morphology data, but there are also wholebody embryo data for different integrative analyses. We provide (1) a developmental atlas for each timepoint; (2) a rigidly aligned and preprocessed $\mu \mathrm{CT}$ scan, dense anatomical landmarks, and segmentations (if available) for each specimen; (3) a set of scripts for transforming and comparing an input scan to an atlas; (4) an approach to validate the transformed landmark data and optimize it, if needed. To ensure reproducibility and data sharing, we make the data freely accessible from FaceBase ${ }^{34}$ 
130 (www.facebase.org, doi.org/10.25550/3-HXMC) (w5 $^{35}$ and our code from GitHub

131 (https://github.com/jaydevine/MusMorph). By incorporating substantial developmental and genetic

132 variation alongside a rich set of metadata, MusMorph will enable standardized morphometric analyses of

133 genotype-phenotypes to better understand the mechanistic basis for morphological variation.

\section{Methods}

135 Mice

136 We compiled mouse embryos and adults from numerous sources. The mouse lines for the E15.5 and E18.5

137 datasets were generated by the IMPC. These mice were produced and maintained on a C57BL/6N genetic

138 background, with support from C57BL/6NJ, C57BL/6NTac or C57BL/6NCrl. More details about

139 husbandry practices can be found at https://www.mousephenotype.org/impress. The mouse lines for the

140 E10.5, E11.5, E14.5, and adult datasets were produced on a variety of genetic backgrounds at different

141 institutions for studies of craniofacial variation. We hereafter refer to these lines as the Calgary mice,

142 because they were ultimately imaged at the University of Calgary. Specific information about study

143 protocols, such as husbandry practices and genotyping, should be gleaned from the MusMorph dataset

144 summaries on FaceBase or the original studies themselves. Each dataset within the MusMorph project on

145 FaceBase represents a study or set of studies defined by a common study design that yielded similar mouse

146 lines. Details about the experimental design were obtained from the original studies listed in the

147 "Publication(s)" section of each dataset. In addition, we provide a supplementary comma-separated values

148 (CSV) file (Study_Metadata.csv) in the project-wide metadata dataset ${ }^{36}$ on FaceBase that lists the associated 149 studies.

150 Micro-computed tomography

151 Sample preparation. Each IMPC embryo underwent a hydrogel stabilization protocol ${ }^{37}$ to prepare for 152 diffusible iodine-based contrast-enhanced $\mu \mathrm{CT}(\text { diceCT })^{38}$. This involved incubating the embryo in a 
hydrogel solution composed of $4 \%$ (wt) paraformaldehyde, $4 \%$ (wt/vol) acrylamide (Bio-Rad, USA), $0.05 \%$ (wt/vol) bis-acrylamide, $0.25 \%$ VA044 Initiator (Wako Chemicals, USA), $0.05 \%$ (wt/vol) saponin (Sigma-Aldrich, Germany), and phosphate-buffered saline at $4^{\circ} \mathrm{C}$ for 3 days. Following incubation, the air in the specimen tube was replaced with nitrogen gas and the tube was immersed in a $37^{\circ} \mathrm{C}$ water bath for 3

$157 \mathrm{~h}$. The whole embryo was then stained with a $0.025 \mathrm{~N}$ to $0.1 \mathrm{~N}$ Lugol's iodine $\left(\mathrm{I}_{2} \mathrm{KI}\right)$ solution (Sigma-

158 Aldrich, Germany) for $24 \mathrm{~h}$ and mounted in agarose for diceCT. This approach has become a popular 159 alternative to magnetic resonance imaging because it is faster, cheaper, and still offers remarkable contrast, 160 allowing for high-throughput phenotyping of soft and hard tissue ${ }^{38}$. acquired prior to 2017 was fixed in a solution of 4\% (wt) paraformaldehyde and 5\% (wt) glutaraldehyde. The specimen was next submerged in the CystoCon Ray II (iothalamate meglumine) contrast agent for one hour to stain external morphology. Embryos obtained after 2017 were put through a nucleic acid stabilization protocol that allows for examination of RNA in embryos scanned via $\mu \mathrm{CT}^{39}$. Each embryo was

176 Imaging. The IMPC embryos were imaged at six centers, including the Baylor College of Medicine, Czech

177 Center for Phenogenomics, MRC Harwell, Toronto Centre for Phenogenomics, The Jackson Laboratory, 
178

179

180

181 182 algorithm ${ }^{40}$.

and University of California, Davis. A 3-D image of each iodine-stained whole embryo was acquired with a Skyscan $1172 \mu \mathrm{CT}$ scanner (Bruker, Kontich, Belgium) at $100 \mathrm{kVp}$ and $100 \mu \mathrm{A}$. The raw images were initially obtained with isotropic voxels but variable spatial dimensions and resolutions, ranging between $0.002 \mathrm{~mm}$ to $0.04 \mathrm{~mm}$. Image projections were reconstructed into a digital stack using the Feldkamp

The Calgary mice were imaged in the 3-D Morphometrics Center at the University of Calgary. A 3-D image of each stained embryo head was obtained with either (a) a Scanco $\mu$ CT 35 scanner (Scanco Medical, Brütisellen, Switzerland) at $45 \mathrm{kV}$ and $177 \mu \mathrm{A}$ or (b) a ZEISS Xradia Versa $520 \mathrm{X}$-ray microscope (Carl Zeiss AG, Oberkochen, Germany) at 40-50 kV, 4-5 W, and 2 s exposure time. A 3-D image of each adult skull was acquired with either (a) a Scanco vivaCT $40 \mu \mathrm{CT}$ scanner (Scanco Medical, Brütisellen, Switzerland), (b) a Scanco vivaCT $80 \mu \mathrm{CT}$ scanner (Scanco Medical, Brütisellen, Switzerland), or (c) a Skyscan 1173 v1.6 $\mu$ CT scanner (Bruker, Kontich, Belgium) at 55-80 kV and 60-145 $\mu$ A. Like the IMPC data, these original images were obtained with isotropic voxels but variable spatial dimensions and resolutions. Embryo image resolutions ranged between $0.007 \mathrm{~mm}$ and $0.027 \mathrm{~mm}$, whereas adult resolutions ranged between $0.035 \mathrm{~mm}$ and $0.044 \mathrm{~mm}$. Image projections were reconstructed with the integrated Scanco software, the ZEISS XMReconstructor software, or the Skyscan NRecon v1.7.4.2 software.

\section{Image preprocessing}

We preprocessed each image to account for differences in image acquisition that would interfere with the atlas-based registration workflow described below (Fig. 1). The preprocessing scripts are provided in the MusMorph GitHub repository (https://github.com/jaydevine/MusMorph/tree/main/Preprocessing). In this preprocessing step, we first converted the reconstructed imaging data (.nrrd, .aim, .tiff) to the Montreal Neurological Institute (MNI) .mnc format using file conversion scripts written in Bash and Python (see AIM_to_MNC.sh, NII_to_MNC.sh, TIFF_to_MNC.sh, DCM_to_MNC.sh, and NRRD_to_MNC.py). As part of the open-source MINC library (http://bic-mni.github.io/man-pages/), the .mnc format is 

internal compression, 64-bit file sizes, and other modern features ${ }^{41}$.

Image preprocessing

\begin{tabular}{|c|c|c|c|}
\hline Stage & E10.5, E11.5, and E14.5 & E15.5 and E18.5 & Adult \\
\hline Preparation & $\begin{array}{l}\text { PFA (4\%) and GD (5\%) } \\
\text { or PAXgene }\end{array}$ & Hydrogel stabilization & Freeze \\
\hline Staining & Cysto or lodine $(1-3.75 \%$ w/v) & lodine $(1-5 \%$ w/v) & None \\
\hline Imaging & $\begin{array}{l}\mu \mathrm{CT} \text { (Scanco } \mu \mathrm{CT} 35 \text { or } \\
\text { ZEISS Xradia Versa } 520 \text { ) }\end{array}$ & $\mu \mathrm{CT}$ (Skyscan 1172) & $\begin{array}{l}\mu \mathrm{CT} \text { (Scanco vivaCT } 40 \\
\text { or Scanco vivaCT } 80 \text { ) }\end{array}$ \\
\hline Preprocessing & $\begin{array}{l}\text { Intensity correction } \\
\text { Intensity normalization } \\
\text { Resample } \\
\text { Initialize }\end{array}$ & $\begin{array}{l}\text { Intensity correction } \\
\text { Intensity normalization } \\
\text { Resample } \\
\text { Initialize }\end{array}$ & $\begin{array}{l}\text { Resample } \\
\text { Initialize }\end{array}$ \\
\hline
\end{tabular}

Image processing and postprocessing

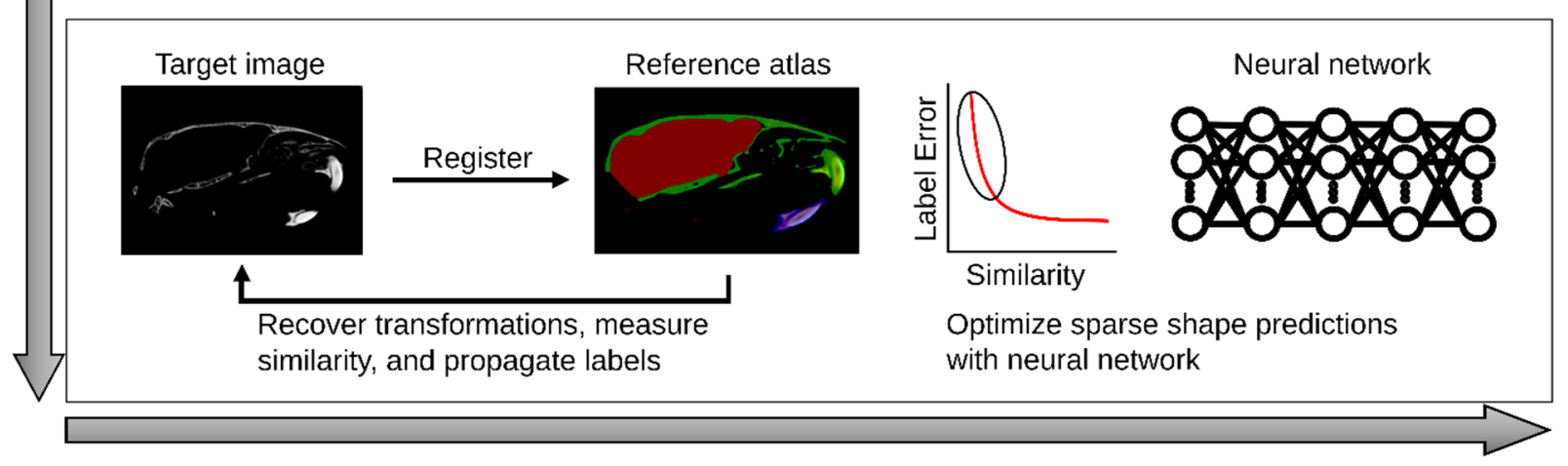

204 Figure 1. Schematic overview of the phenotyping pipeline. Specimens were staged, prepared (fixed/stored), stained, and imaged with different but standardized lab-specific protocols. While the E10.5, E11.5, E14.5, and adult specimens were obtained in Calgary, the E15.5 and E18.5 specimens were acquired from the IMPC. To account for differences in image acquisition (e.g., intensity artifacts, image resolution and dimensions, and position), each image was subjected to a series of preprocessing steps. Next, each preprocessed image was non-linearly registered to a stage-specific reference atlas with a detailed set of landmarks and segmentations. We recovered deformation fields, landmarks, and segmentations (if available) for each specimen. To optimize the landmark predictions of poorly registered specimens, as measured by cross-correlation similarity, a downstream neural network was used.

214 registration process. To minimize intensity inhomogeneities, we applied the N3 method ${ }^{42}$. Since many of

215 the E15.5 images had background noise, where the stained scanning medium was indistinguishable from

216 the anatomy, we employed a thresholding script in Bash (see Threshold.sh). This script computes a lower 
anatomical density threshold, masks the voxels above this bound and those in proximity via dilation, and equates all voxels outside the mask to 0 . To ensure the image resolutions and dimensions were consistent with the atlas, we implemented an image resampling script in Bash (see Downsample_and_Correct.sh). We also used this script to control for differences in bit depth among scanners by including a min-max normalization, which scaled the embryo intensities between 0 and 1 . Table 1 outlines the source of the image data, developmental stage, voxel dimensions, image resolutions, stage-specific sample sizes, and the presence or absence of atlas anatomical labels. Note that the E14.5 images were solely used to create another

224 stage-specific atlas, as they are from a smaller, unpublished dataset.

Table 1. Summary of imaging data. Source is where the image was acquired. Stage is the age of the specimen at sacrifice. Anatomy is the labelled and scanned anatomy. X, Y, and Z are the voxel lengths of each atlas axis. Resolution is the isotropic resolution of each scan. $\mathrm{N}$ is the sample size, with the number of scans awaiting publication of primary research in parentheses. Landmarks and segmentations indicate the presence $(\checkmark)$ or absence $(x)$ of labels on the stage-specific atlas.

\begin{tabular}{|l|l|l|l|l|l|l|l|l|l|}
\hline Source & Stage & Anatomy & $\mathbf{X}$ & $\mathbf{Y}$ & $\mathbf{Z}$ & $\begin{array}{l}\text { Resolution } \\
(\mathbf{m m})\end{array}$ & $\mathbf{N}$ & $\begin{array}{l}\text { Landmark } \\
\text { s }\end{array}$ & Segmentations \\
\hline Calgary & E10.5 & Head & 220 & 295 & 350 & 0.012 & 434 & $\checkmark$ & $\times$ \\
\hline Calgary & E11.5 & Head & 502 & 503 & 390 & 0.012 & 531 & $\checkmark$ & $\times$ \\
\hline Calgary & E14.5 & $\begin{array}{l}\text { Head; } \\
\text { Body }\end{array}$ & 486 & 567 & 723 & 0.027 & $\begin{array}{l}84 \\
(84)\end{array}$ & $\checkmark$ & $\times$ \\
\hline IMPC & E15.5 & $\begin{array}{l}\text { Head; } \\
\text { Body }\end{array}$ & 486 & 567 & 723 & 0.027 & 1426 & $\checkmark$ & $\checkmark$ \\
\hline IMPC & E18.5 & $\begin{array}{l}\text { Head; } \\
\text { Body }\end{array}$ & 293 & 414 & 667 & 0.054 & 1657 & $\checkmark$ & $\times$ \\
\hline Calgary & Adult & $\begin{array}{l}\text { Skull } \\
\text { Skeng }\end{array}$ & 642 & 586 & 979 & 0.035 & $\begin{array}{l}6000 \\
(154)\end{array}$ & $\checkmark$ & $\checkmark$ \\
\hline
\end{tabular}

227 between an image pair. Using initialization scripts written in Bash (see Preprocessing.md) and R 228 (Tag_Combine.R), we rigidly transformed each image to a stage-specific atlas or, if an atlas did not exist, 229 an arbitrary but stage-specific reference image. To determine the rigid transformation matrices, we utilized 230 a manual and automated approach, or a strictly automated approach, depending on anatomical orientation.

231 If the mouse was scanned in a random orientation, we rendered a minimum threshold surface in MINC, 
then manually placed five homologous three-dimensional (3-D) landmarks at anatomical extrema (e.g., ears, nose, top of the head, and back of the head), resulting in an MNI tag point file (.tag) with landmark

234 coordinates. Next, we concatenated the reference and arbitrary landmark matrices, and minimized their 3-

235 D Euclidean distances via least squares. If the specimen was already roughly aligned to the reference image,

236 we performed an automated, intensity-based rigid alignment using the full registration process outlined

237 below (see the "Image Registration and Label Propagation" section). This intensity-based rigid alignment

238 was also repeated for the manually aligned volumes to ensure consistency. With the rigid transformation

239 matrices, we resampled each image into their stage-specific reference coordinate space using tri-linear

240 interpolation.

\section{Reference atlases}

242 We generated a population average atlas for each stage, excluding E15.5 and adulthood, by spatially 243 normalizing $25 \mu \mathrm{CT}$ images of wildtype mice with a group-wise registration workflow ${ }^{43,44}$ (Fig. 2 and 3).

244 A nearly identical workflow was used to create the existing E15.5 and adult atlases. The atlas construction

245 script is available in the MusMorph

246 (https://github.com/jaydevine/MusMorph/tree/main/Processing) and is written in Python (see

247 HiRes_Atlas.py or LoRes_Atlas.py). This script produces Bash scripts that can be executed automatically

248 and in parallel on a compute cluster to maximize computational efficiency. Without massively parallel

249 computing, the volumetric registrations would need to be performed sequentially, each requiring hours of

250 computation and a large amount of memory. Before executing the workflow, the user must upload the

251 initialized images and registration scripts to a compute cluster. In addition, the user needs to install a MINC

252 Toolkit module onto the cluster via Docker (https://bic-mni.github.io/) or GitHub (https://github.com/BIC-

$253 \mathrm{MNI} /$ minc-toolkit-v2), or define a pre-existing module, because the scripts utilize the open-source MINC

254 software. An atlas can also be generated locally, but it will be significantly slower without massively 255 parallel computing. 
bioRxiv preprint doi: https://doi.org/10.1101/2021.11.11.468142; this version posted November 12, 2021. The copyright holder for this preprint

(which was not certified by peer review) is the author/funder, who has granted bioRxiv a license to display the preprint in perpetuity. It is made

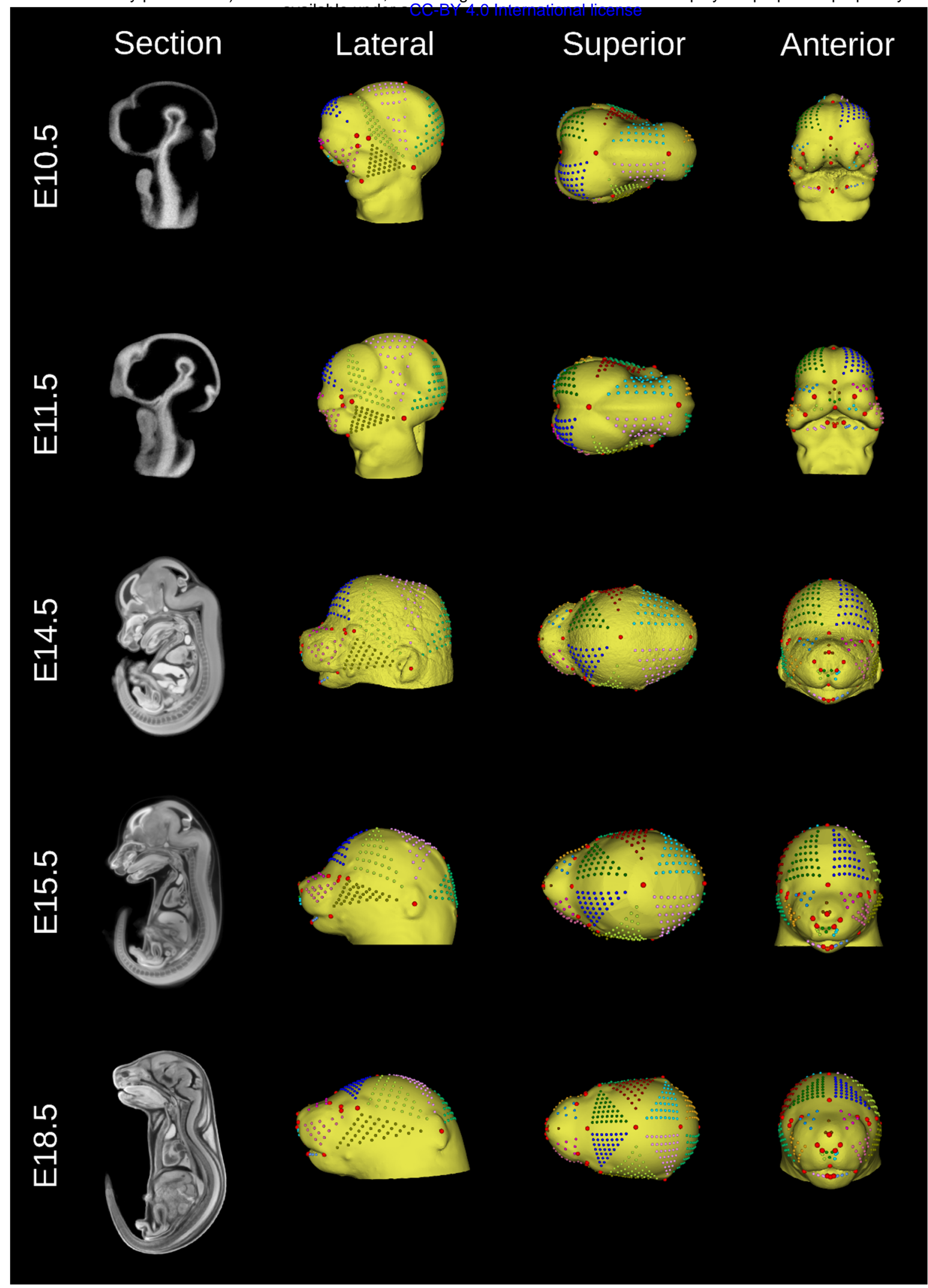

Figure 2. Embryo reference atlases. Sagittal cross-sections of the E10.5 (www.facebase.org/id/6F00W), E11.5 (www.facebase.org/id/6-F012), E14.5 (www.facebase.org/id/6-F016), E15.5 (www.facebase.org/id/6-F6SE), and E18.5 (www.facebase.org/id/6-F6T4) atlas volumes are shown to display the stained internal anatomy. Each head surface was labelled with a dense landmark configuration to capture global and local aspects of morphology. Lateral, superior, and anterior views of each head isosurface are shown. The semi-landmark patches (small, color-coded points) were interpolated between a set of sparse homologous landmarks (large, red points). They can be slid and resampled for morphometric analyses. 

deformable transformation for non-linear alignment. To account for global variation in location, orientation, and scale, we computed a series of multi-resolution (coarse to fine) affine transformations among the

260 images by optimizing a cross-correlation objective function ${ }^{45}$. Given that sample-wide pairwise 261 registrations yield an improved affine template ${ }^{46}$, or intensity average, we completed all possible $\left(N=25^{*} 24\right)$

262 pairwise affine registrations, then averaged the resulting transformation for each specimen. Using the 263 averaged transformations, we resampled each initialized image into the affine coordinate space with tri264 linear interpolation and averaged the resulting images to produce an affine template. To correct for local 265 variation in shape, we computed a series of multi-resolution non-linear transformations with the ANIMAL 266 (Automatic Nonlinear Image Matching and Anatomical Labelling) algorithm ${ }^{47}$, again optimizing for cross267 correlation. This iterative, four-step process involves non-linearly deforming each mouse to an evolving template at increasingly higher resolutions, with the first template being the affine average and the next three being improved versions of the non-linear average $e^{48}$. The final product is a stage-specific average with excellent contrast and a high signal-to-noise ratio.

272 imaging data are head scans, we focused on labelling each atlas head surface with a standardized landmark 273 configuration (Fig. 2 and 3). Specific information about the number of landmarks and their anatomical 274 definitions can be found below in the "Data Records: Landmarks" section. To generate the landmarks, we 275 first rendered a minimum density isosurface in MINC, which uses ITK's marching cubes algorithm, and 276 saved the 3-D rendering as a Stanford PLY (.ply) file. We then used 3D Slicer ${ }^{49}$ or the MINC Toolkit to 277 acquire a landmark configuration on each surface that provided a comprehensive representation of shape ${ }^{50}$. 278 For the embryos, we used 3D Slicer and the SlicerMorph extension ${ }^{32}$ to identify sparse landmarks and 279 interpolate landmark patches of variable density in between, depending on the size of the area, resulting in 280 dense coverage of the head. This also ensured that the patches were homologous, allowing for a 281 developmental morphospace into which all specimens may be superimposed. Because developmental 


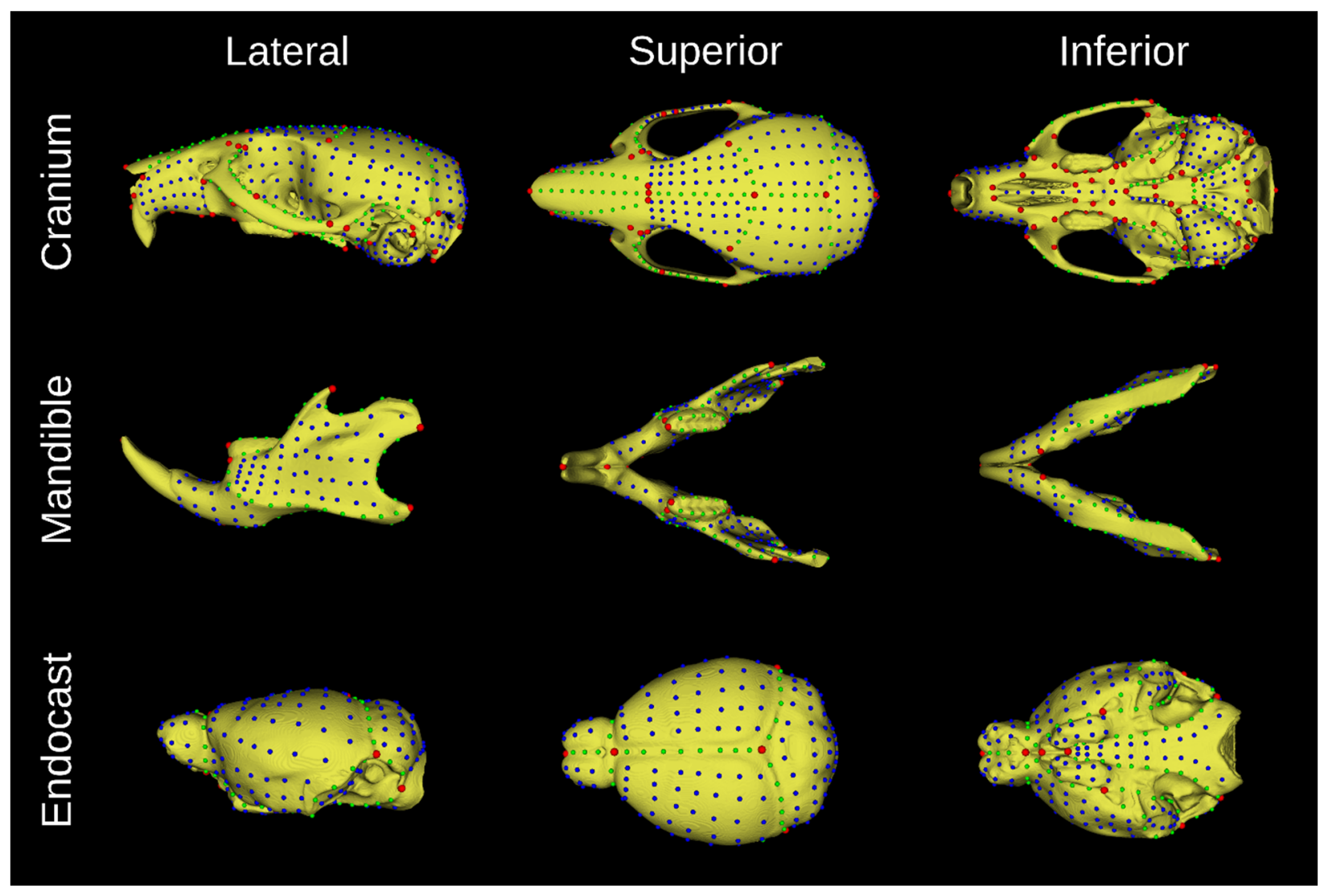

Figure 3. Adult reference atlas. Cranium (top), mandible (middle), and endocast (bottom) surfaces were segmented from the skull atlas (www.facebase.org/id/6-F6VC), then labelled with a dense landmark configuration to capture global and local aspects of morphology. Lateral, superior, and anterior views of each segmentation isosurface are shown. There are sparse landmarks (red) as well as surface (blue) and curve (green) semi-landmarks that can be slid and resampled for morphometric analyses.

Shared developmental pathways lead to correlated morphological variation, or morphological

290 integration ${ }^{51-57}$. To enable analyses of integration, we added landmark configurations to segmented surfaces

291 of the adult skull atlas. We manually segmented the cranium, mandible, and neurocranial endocast (i.e., a

292 proxy for the brain) in MINC, then rendered these segmentations as isosurfaces before landmarking them

293 with a dense configuration. Once again, the landmark details are described below in the "Data Records:

294 Landmarks" section. The segmentations may further be used for surface-based analyses ${ }^{58}$, measures of size

295 (e.g., volume or surface), or as masks to reduce the shape dimensionality of a voxel-based morphometry

296 analysis. Unlike the adult atlas, the embryo atlases do not come with segmentations due to the scope of this 
work, apart from the pre-existing E15.5 atlas, which has 48 manually segmented structures

298 (http://www.mouseimaging.ca/technologies/mouse_atlas/mouse_embryo_atlas.html).

We pairwise registered each image to their stage-specific atlas to obtain a composite (affine and non-linear) transformation for label propagation (Fig. 1). Like the atlas workflow described above, the registration scripts are available

in the MusMorph

GitHub

303 (https://github.com/jaydevine/MusMorph/tree/main/Processing) and are written in Python (see HiRes_Pairwise.py or LoRes_Pairwise.py). The purpose once more is to produce Bash scripts en masse for massively parallel computing on a compute cluster due to the computational requirements of volumetric deformable registration and anatomical labelling. Only the initialized images and registration scripts need to be uploaded to the cluster to execute the workflow. While the pairwise registrations involved the same multi-resolution affine alignment described above, the non-linear alignment differed. Here, we implemented the geodesic SyN (Symmetric Normalization) algorithm ${ }^{59}$, because it was previously

310 validated for atlas-based landmarking and morphometrics of mouse models ${ }^{44}$. The SyN registrations were

311 optimized using cross-correlation. After registration, we used labelling scripts written in Bash and produced

312 via Python (see Label_Propagation.py) to recover the non-linear transformations, concatenate them with

313 the affine transformations, invert them, and propagate the atlas labels to the rigid space of each image.

\section{Neural network shape optimization}

315 Although top-performing registration algorithms provide an effective and generalizable way to

316 automatically label anatomy, there are instances where outliers and problematic landmarks can alter shape

317 representations. This is particularly true for model organisms, where mutant phenotypes may show little to

318 no resemblance with an atlas. To demonstrate how biological signal can be restored, we implemented a

319 supervised deep learning workflow available in the MusMorph GitHub

320 (https://github.com/jaydevine/MusMorph/tree/main/Postprocessing), which employs scripts written in $\mathrm{R}$ 
321 and Julia (see GPA_and_Projection.R and Landmark_Optimization.jl) ${ }^{60}$. Using a subset of 68 sparse adult

322 craniofacial landmarks $(N=2,000)$ described in previous work ${ }^{61-65}$, we trained a deep feedforward neural

323 network to learn a domain-specific loss function that minimizes automated and manual shape differences.

324 The sparse landmark numbers amenable to optimization (see Optimization_Order.csv) ${ }^{36}$ are available on

325 FaceBase. We focused on the adults because that was the only stage with a large existing set of homologous

326 manual landmarks for training.

327 We tested the network predictions on a random subset $(N=500)$ of adult skulls described further in

328 the "Technical Validation" section. To help others initialize the network without having to retrain it, we 329 provide the adult network model (Calgary_Adult_Cranium_Model.bson) and weights

330 (Calgary_Adult_Cranium_Weights.bson) in the Binary JSON (.bson) file format on GitHub. We also make 331 available the optimized sparse shape predictions for the entire adult crania dataset

332 (Adult_Cranium_Sparse_Landmarks.csv) ${ }^{36}$. Although we focused on adults, this optimization strategy is 333 generalizable, so other research groups with manual landmark data on any structure of the atlases may use 334 the network architecture to improve outlier predictions.

\section{Data Records}

\section{Specimen metadata}

337 Each specimen is associated with a rich set of identifiers to accommodate morphometric analyses using 338 multiple factors and/or covariates. Alongside detailed metadata descriptions in FaceBase, we provide the 339 specimen metadata as a supplementary CSV file (MusMorph_Metadata.csv) ${ }^{36}$ for convenience and to 340 include auxiliary fields. Table 2 enumerates the metadata and Table S1 summarizes the metadata 341 distributions for each dataset on FaceBase. 
Table 2. Summary of metadata identifiers.

\begin{tabular}{|l|l|}
\hline Identifier & Description \\
\hline Biosample & The name of the specimen, which corresponds to the image and label names. \\
\hline Strain & The background strain of the specimen. \\
\hline Strain_MGI_ID & The MGI ID for the strain. \\
\hline Strain_Type & $\begin{array}{l}\text { An attribute of strain that describes whether it is inbred or outbred and lab-derived } \\
\text { or wild-derived. }\end{array}$ \\
\hline Gene & The gene symbol as provided by MGI. \\
\hline Gene_MGI_ID & The MGI ID for the gene. \\
\hline Zygosity & $\begin{array}{l}\text { Whether the specimen is homozygous, heterozygous, wildtype, or otherwise (e.g., } \\
\text { flox/null) for a given gene mutation. }\end{array}$ \\
\hline Genotype & A concatenation of the gene symbol and zygosity symbol. \\
\hline Anatomy & The region of anatomy that has been scanned and labelled. \\
\hline Treatment & An environmental effect that the specimen has been treated with. \\
\hline $\begin{array}{l}\text { Experimental } \\
\text { Group }\end{array}$ & $\begin{array}{l}\text { An identifier derived from genotype that denotes whether the specimen is a control } \\
\text { or mutant. }\end{array}$ \\
\hline Sex & The sex of the specimen. \\
\hline Stage & The age of the specimen in days, either embryonic (E) or postnatal (PN). \\
\hline Life_Phase & $\begin{array}{l}\text { An identifier derived from stage that indicates life phase (e.g., gestation vs. } \\
\text { adulthood). }\end{array}$ \\
\hline Dataset & $\begin{array}{l}\text { The published or unpublished study (see Study_Metadata.csv) the sample is } \\
\text { associated with. }\end{array}$ \\
\hline Availability & $\begin{array}{l}\text { Whether the images and phenotypic data are available or pending publication of a } \\
\text { primary research article. }\end{array}$ \\
\hline
\end{tabular}

Fig. 4a-b illustrates the distributions of sex, strain type, and genotype across the embryo and adult

344 datasets. Sex is well-annotated for the E15.5, E18.5, and adult datasets, but is missing ("NA") for many of

345 the E10.5 and E11.5 specimens. While most of the embryo mouse models were produced on an isogenic

346 inbred background, particularly C57BL/6N, strain diversity is a focal point of the adult datasets. Among

347 the nine adult strain types provided, there are 98 unique background strains. The majority are recombinant 348 inbred lines (e.g., the Collaborative Cross dataset ${ }^{66}$ ), wild-derived crosses (e.g., the Hybrid dataset ${ }^{67}$ ), and 349 outbred lines (e.g., the Diversity Outbred dataset ${ }^{68}$ ). We have included 459 unique genotypes for the embryo 350 datasets, most of which derive from the IMPC dataset ${ }^{69}$, as well as 179 genotypes for the adult datasets. A 351 minority of specimens, including several embryos in the $\mathrm{Ap} 2^{70}, \mathrm{~B} 9 \mathrm{~d}^{71}$, and Bulgy ${ }^{72}$ datasets as well as a 352 few adults in the Brain-Face ${ }^{73}$ dataset, have unknown genotypes (e.g., "-/-;NA" and "+/-;NA" in double 
bioRxiv preprint doi: https://doi.org/10.1101/2021.11.11.468142; this version posted November 12, 2021. The copyright holder for this preprint (which was not certified by peer review) is the author/funder, who has granted bioRxiv a license to display the preprint in perpetuity. It is made

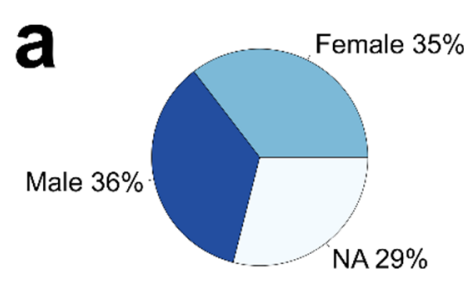
available under aCC-BY 4.0 International license.
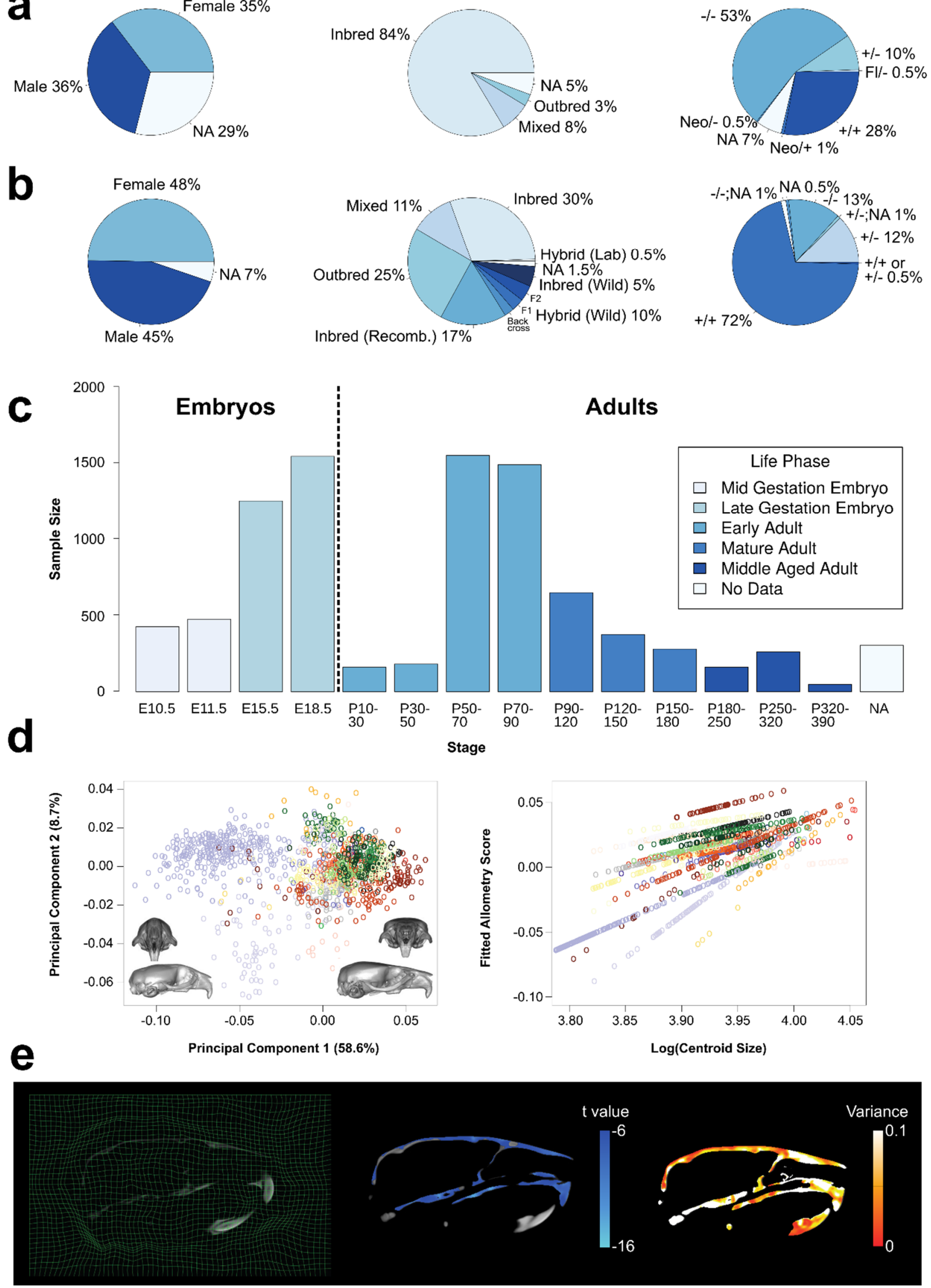

Figure 4. Summary of metadata. (a) Distribution of sex, strain type, and genotype for the embryo datasets. (b) Distribution of sex, strain type, and genotype for the adult dataset. (c) Sample sizes of each developmental stage included in the database. All "NA" specimens are mature or middle-aged adults. (d) Left: Example landmarks and segmentations of the adult skull and endocast (brain). Middle/Right: Morphological analyses, such as PCA and allometry regressions, that one might perform with a dense landmark dataset. Each color in the plot represents a different mouse genotype. 
(e) Left: Slice visualization of a non-linear deformation grid. Middle/Right: Morphological analyses, such as statistical parametric mapping, that one might perform with a deformation field. The $t$ values show significant $(\mathrm{p}<0.05)$ voxel-wise differences in form (i.e., volume shrinkage) in Ghrhr homozygous mutants relative to wild type, whereas the variance heatmap shows voxel-wise variances in Ghrhr mutants.

354 knockout designs or "NA" and "+/+ or +/-" in single knockouts) due to genotyping complications in the

355 past. Specimens homozygous for a single gene mutation predominate the embryo datasets, whereas normal

356 wildtype variants comprise the bulk of the adult datasets. Fig. 4c shows the developmental stages

357 represented in MusMorph. Of the 10,056 specimens processed, $40 \%$ are embryos and $60 \%$ are adults, many

358 of which have just finished maturing around postnatal day 90. All specimens without a recorded stage

359 ("NA") are mature adults.

It is often desirable to compare mutants to their wildtype counterparts from the same sample

361 because background strains vary. To preserve sample provenance where possible, specimens that are

362 wildtype for a given mutation will have the same gene symbol as their heterozygote and homozygote

363 littermates. For wildtype specimens without litter information, like the IMPC dataset, their genotypes are

364 equated to background strain. Mouse strain nomenclature follows the MGI guidelines, except when the

365 strain design is unknown and has no MGI ID (e.g., novel hybrid backcrosses). We also abbreviate genotypes

366 for complex strain designs using MGI synonyms if available. Furthermore, while most wildtype specimens

367 fall within the control experimental group, there are cases where they can exhibit mutant-like phenotypes

368 and be categorized as such. One example in MusMorph is the artificial selection Longshanks dataset ${ }^{74}$,

369 which through many generations of artificial selection produced wildtype specimens with extreme tibia and $370 \quad$ craniofacial phenotypes ${ }^{75,76}$.

371 We selected the above identifiers, because they tend to explain a significant amount of 372 morphological variation in morphometric analyses. For instance, many structures in the mouse are sexually 373 dimorphic, including the shape of the $\operatorname{brain}^{77}$ and craniofacial complex ${ }^{78}$, cortical bone size and strength ${ }^{79}$, 374 adipose tissue distribution ${ }^{80}$, and feto-placental growth ${ }^{81,82}$, to name a few. It is also known that classical 375 laboratory strains, such as those in the Strain Comparison dataset ${ }^{83}$, exhibit naturally occurring craniofacial 
phenotypes $^{84}$. Moreover, gene mutations can interact with a background strain via epistasis to produce different phenotypes ${ }^{85-87}$, like those in the Spry dataset ${ }^{88}$. Another key driver of variation is developmental stage, as differences in age often define a principal axis of allometric variation via correlations with size and/or shape ${ }^{89-93}$. Given the ubiquity of allometry, these correlations can be found across most MusMorph datasets (Fig. 4d). Finally, numerous studies have reported the phenotypic outcomes of single gene mutations, environmental perturbations, and how zygosity modulates these effects ${ }^{94-96}$. These identifiers have corresponding images, landmarks, segmentations, and deformation fields for morphological analyses (Fig. 4d-e).

\section{Images}

We provide the atlases and initialized images for each specimen in the MNI .mnc format. The naming

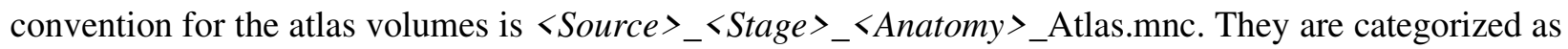

387 "Imaging Data" in the project-wide dataset ${ }^{36}$ on FaceBase. The naming convention for the initialized 388 volumes is 〈Biosample>.mnc, where Biosample is the name of the specimen in the metadata (see the 389 "Specimen metadata" section). One exception is the naming convention for the subset of thresholded E15.5 390 images, which is <Biosample〉_Thresh.mnc. These volumes are also categorized as "Imaging Data" across 391 the MusMorph datasets on FaceBase. Each .mnc file has four key attributes: 1) a named dimension (xspace, yspace, zspace), 2) length (number of voxels on each dimension), step (resolution), and start (origin). MINC defines a voxel and world coordinate system, so one can move between them with the simple "voxeltoworld" and "worldtovoxel" MINC commands. If users want to convert between .mnc and different

\section{Transformations}


400 For each pairwise registration, we recovered an inverted non-linear and composite (affine and non-linear)

401 transformation. Given the file sizes of the non-linear deformation fields $(\sim 3 \mathrm{~GB}$ on average $\times 10,000=30$

402 TB), we make the transformations available upon request. The deformation fields and composite

403 transformations are in the MNI .mnc and .xfm formats. Each .mnc file shares the same image attributes

404 described above with an additional named dimension called vector_dimension which describes the non-

405 linear displacement vectors. Each .xfm file contains a header and affine transformation matrix. The naming

406 convention for the deformation fields is <Biosample〉_ANTS_nl_inverse_grid_0.mnc and

407 <Biosample>_ANTS_nl_inverse.xfm, whereas the composite transformations are called

408 <Biosample>_origtoANTSnl_grid_0.mnc and <Biosample>_origtoANTSnl.xfm. "ANTS" denotes the

409 algorithm and "nl" stands for "non-linear". Much like the images, the transformations for the subset of

410 thresholded E15.5 volumes have "Thresh" appended to the <Biosample $>$ name.

412 reference image voxel ${ }^{97}$. By calculating the Jacobian determinant $J$ for every point $p(x, y, z)$ in the 413 deformation field,

$$
\operatorname{det}(J(x, y, z))=\left|\begin{array}{lll}
\frac{\partial x}{\partial x} & \frac{\partial y}{\partial x} & \frac{\partial z}{\partial x} \\
\frac{\partial x}{\partial y} & \frac{\partial y}{\partial y} & \frac{\partial z}{\partial y} \\
\frac{\partial x}{\partial z} & \frac{\partial y}{\partial z} & \frac{\partial z}{\partial z}
\end{array}\right|
$$

415 one can quantify the magnitude of morphological change at each voxel (Fig. 4e). A Jacobian determinant

416 of 1 indicates no volume change, whereas determinants greater than 1 indicate volume expansion and

417 determinants between 0 and 1 indicate volume shrinkage. These determinants can also be scaled and

418 sheared with a composite transformation to examine voxel-wise differences in form. Jacobian determinants

419 can be analyzed with voxel-wise tests, such as an ANOVA with a false-discovery rate correction, to map

420 statistics onto the anatomy, a technique otherwise known as statistical parametric mapping (see 
421 VBM_Example.R). For example, in Fig. 4e, we use the RMINC R package (https://github.com/Mouse-

422 Imaging-Centre/RMINC) to show significant voxel-wise changes (shrinkages) in form between Ghrhr

423 mutants $^{98}$ and wildtype specimens, as well as voxel-wise variances in form associated with this mutation.

\section{Landmarks}

We labelled each atlas, and thus every registered mouse embryo and adult, with a standardized landmark

files are named $\langle\text { Source }\rangle_{-}\langle$Stage $\rangle$<Anatomy $\rangle$Atlas_Landmarks.tag. They are stored as "Imaging Data" alongside the atlas volumes on FaceBase ${ }^{36}$. The individual specimen landmark files are named <Biosample>_<Anatomy>_Landmarks.tag and are similarly categorized as "Imaging Data" across FaceBase. The MNI .tag file format is an ASCII file which stores the coordinates of each landmark in the millimetric world space of the volume. Each tag file has a header above an array of $p$ landmarks (rows) in $k$ dimensions (columns). These files can be imported into $\mathrm{R}$ individually or collectively as a 3-D array using the tag2array function in the custom morpho.tools.GM package ${ }^{99}$. Alternatively, the user can employ the read.csv function in $\mathrm{R}$ to import a vectorized .csv file. We provide landmark .csv files for every developmental stage and anatomical region ${ }^{36}$, each of which contains a matrix of $n$ specimens (rows) and $p \times k$ landmark coordinate dimensions (columns). Importantly, there are dense semi-landmarks and sparse

437 fixed landmarks for local and global geometric morphometric analyses of craniofacial, endocast (brain), 438 and mandible morphology. In Fig. 4d, for instance, we show craniofacial shape morphs along the first 439 principal component (PC) in an adult subsample, as well as allometry regressions which relate craniofacial $440 \quad$ shape to size.

The embryo landmarks are homologous across stages. Table S2 describes the sparse embryo

442 landmarks and their biological definitions. Table S3 lists the embryo semi-landmark patches and their 443 density, both of which are based on the sparse landmarks. The stage-specific semi-landmark patch files can 444 also be found as tab-separated value (TSV) files on GitHub 

homologous landmarks within their larger dense configuration. To perform a sparse landmark shape

447 analysis, users may subset the first 22 rows of each 3-D array. Since there are three additional sparse 448 landmarks for the E15.5 and E18.5 specimens, rows 23 to 25 may be included for stage-specific analyses 449 or excluded for ontogenetic analyses. adult craniofacial, endocast, and mandible landmarks, respectively, as well as their biological definitions. While the adult curve semi-landmarks and surface semi-landmarks are not patch based, they can be slid and resampled using the $\mathrm{R}$ scripts on GitHub (see Calgary_Adult_Cranium_Sliding_Semis.R, rows of the cranium, endocast, and mandible 3-D arrays, respectively, and can be partitioned for a sparse shape analysis. If users want to generate new landmarks, such as internal landmarks or whole-body landmarks, they can use a script (see Label_Propagation.py), the inverted composite transformations (see the "Transformations" section), and a local or remote compute cluster to propagate the landmarks to an 460 initialized image. To promote standardization, we encourage users to add new landmark subsets to the pre461 existing configurations.

\section{Segmentations}

463 We provide segmentation labels for the E15.5 and adult atlases and specimens to support alternative 464 morphological analyses, such as 3-D visualizations, voxel-based morphometry, volumetric size 465 comparisons, and surface-based image processing pipelines. Other stages do not have segmentation labels 466 due to the scope of this work. The segmentations follow the same naming conventions described above:

$467\langle$ Source $>$ $\langle$ Stage $\rangle$ Atlas_Segs.mnc and $\langle$ Biosample $\rangle$ Segs.mnc. The atlas segmentations are available 468 as "Imaging Data" on FaceBase ${ }^{36}$, as are the individual segmentation files across various MusMorph 
datasets. The

published

E15.5 atlas

contains

48

whole body

segmentations

470 (http://www.mouseimaging.ca/technologies/mouse_atlas/mouse_embryo_atlas.html ${ }^{48}$, while the adult

471 atlas comes with cranium, endocast, and mandible segmentations. Each label file is a .mnc volume of

472 integers that matches the dimensionality of the image. To visualize the adult segmentations, for example,

473 the user may load the atlas and label files together and input an integer of 1 to render the endocast, 2 for the

474 cranium, and 3 for the mandible. As with new landmarks, there is the potential to resample new atlas

475 segmentation labels into the initialized space of any image using the composite transformations (see the

476 "Transformations" section) and a local or remote compute cluster (see Label_Propagation.py).

\section{Technical Validation}

\section{Cross-correlation and root mean squared error}

479

480

481

We computed intensity-based, pairwise registrations between each target image $(I)$ and a reference atlas $(J)$ by optimizing a normalized cross-correlation (NCC) similarity metric:

$$
\operatorname{NCC}(I, J)=\frac{\sum_{p \in \Omega} I_{(p)} J_{(p)}}{\sqrt{\sum_{p \in \Omega} I_{(p)}{ }^{2} \sum_{p \in \Omega} J_{(p)}{ }^{2}}} .
$$

Normalized cross-correlation is calculated for all voxel positions $p$ over a discrete domain $(p \in \Omega)$. If the domain is the entire 3-D volume and $\operatorname{NCC}(I, J)=1$, the deformed target image and reference image are perfectly aligned. To assess the quality of each registration, we recorded the normalized cross-correlation between each deformed target image and the atlas using code in the labelling scripts (see Label_Propagation.py). Unfortunately, it is difficult to know whether the final registration correlations are "good" or "bad" without relating them to the quality of the labels collected. We investigated the relationship between landmark root mean squared error and cross-correlation in the adult crania training set above to build a quality assessment model. Letting $\boldsymbol{x}_{\boldsymbol{\ell}}^{(I)}$ and $\widehat{\boldsymbol{x}}_{\boldsymbol{\ell}}^{(I)}$ denote the observed (manual) and predicted 
490 (automated) Euclidean vectors at landmark $\ell$ for a target image $I$, the root mean squared error for $p$

491 landmarks is defined as

$$
R M S E=\sqrt{\sum_{\ell=1}^{p} \frac{1}{n} \sum_{I=1}^{n}\left(\boldsymbol{x}_{\boldsymbol{\ell}}^{(I)}-\widehat{\boldsymbol{x}}_{\boldsymbol{\ell}}^{(I)}\right)^{2}}
$$

After computing the root mean squared error for each specimen, we regressed these values on their corresponding cross-correlation values with linear, squared, and cubic cross-correlation terms (Fig. 5a). We

495 found a statistically significant non-linear relationship $\left(R^{2}=0.3, \mathrm{p}<0.001\right)$, such that cross-correlation 496 values below 0.90 resulted in exponentially higher landmark errors. The average root mean squared error 497 was $0.23 \mathrm{~mm}(95 \% \mathrm{CI} \pm 0.002 \mathrm{~mm})$. This mean error is comparable to manual landmark intra-observer 498 detection errors across the skull, which tend to be $0.25 \mathrm{~mm}$ or less ${ }^{44,50}$. To verify registration quality across the rest of the database, we calculated cross-correlations for all specimens and stages. The mean crosscorrelation values and their standard deviations for E10.5, E11.5, E15.5, E18.5, and adulthood were $0.94 \pm$ this approach for analyzing variable morphology.

\section{Covariance patterns and the mean shape}

505 We quantified differences in covariance structure and the sample mean shape between our baseline automated landmarks, the optimized neural network landmarks, and the manual landmarks. To analyze covariance similarity, we projected the automated configurations into the manual PC space and correlated the uncentered PC scores. Fig. 5b shows automated and manual correlations for the first 10 PCs $(65.1 \%$ of

509 the total variance). The average correlation within PCs for the baseline automated configurations was $r=$

510 0.6. This measure is biased downwards by lower order automated PCs, which tend to capture residual

511 covariance of the first manual PC. The average correlation within PCs for the optimized automated 512 configurations was $r=0.8$, suggesting a restoration of signal among the major PCs. 
bioRxiv preprint doi: https://doi.org/10.1101/2021.11.11.468142; this version posted November 12, 2021. The copyright holder for this preprint (which was not certified by peer review) is the author/funder, who has granted bioRxiv a license to display the preprint in perpetuity. It is made available under aCC-BY 4.0 International license.

a

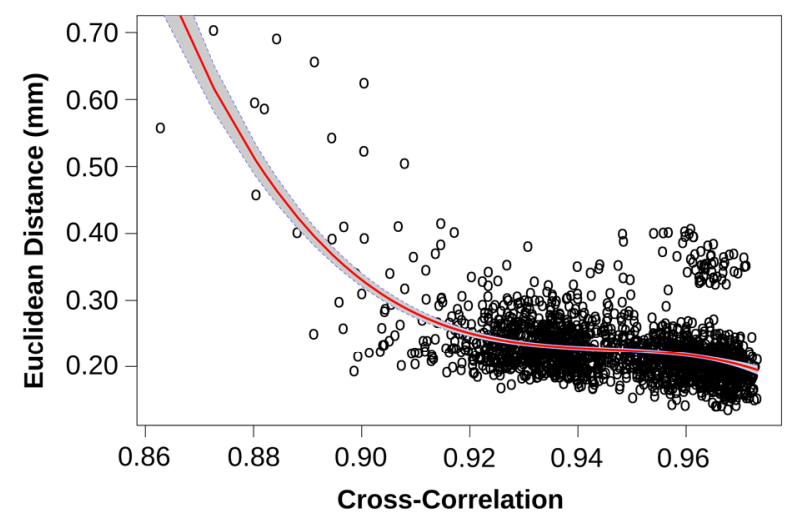

b

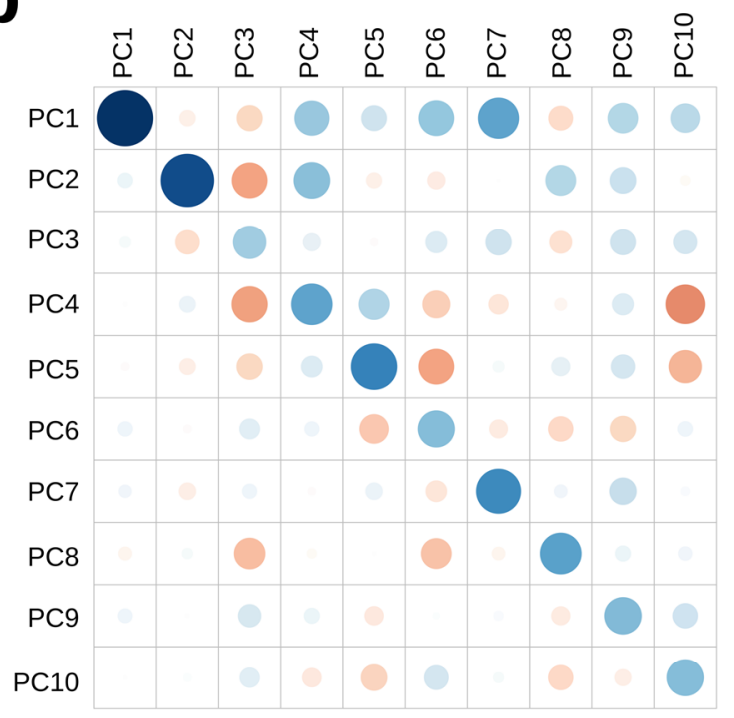

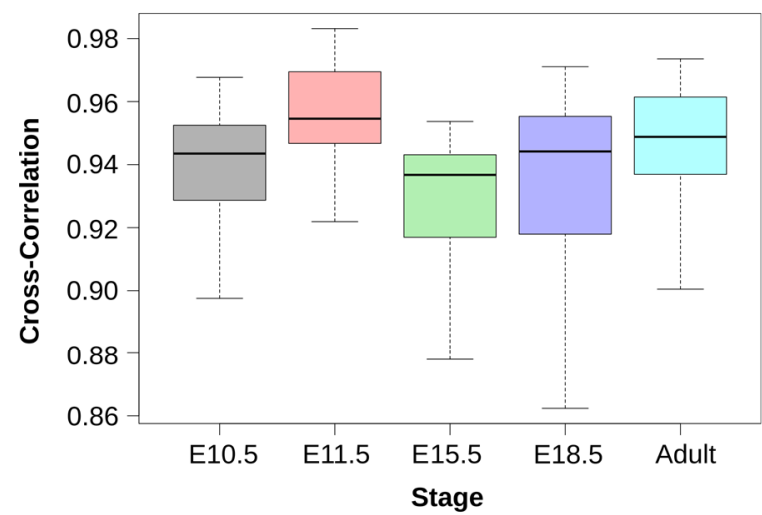

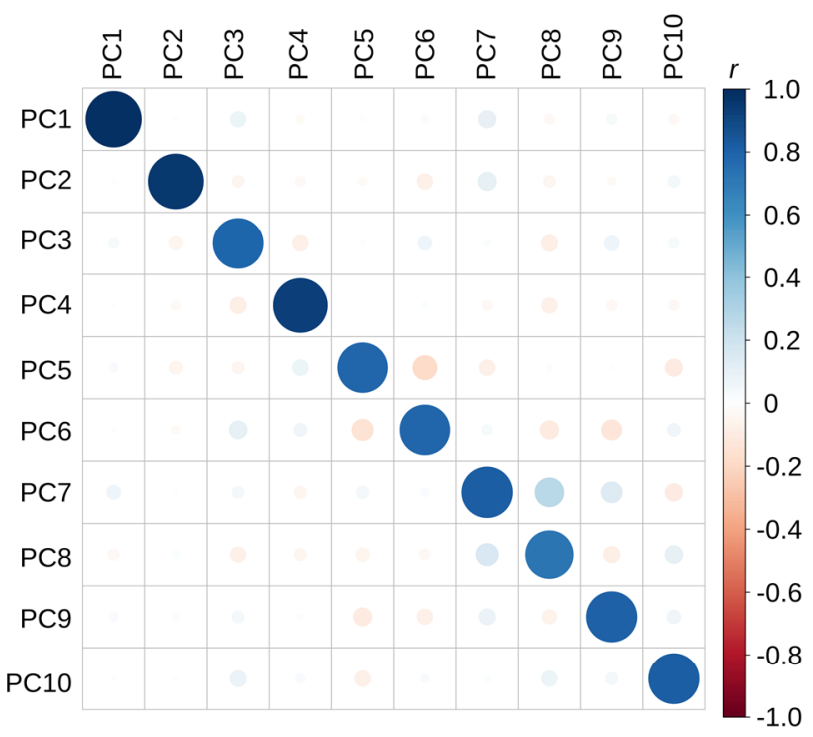

\section{C}

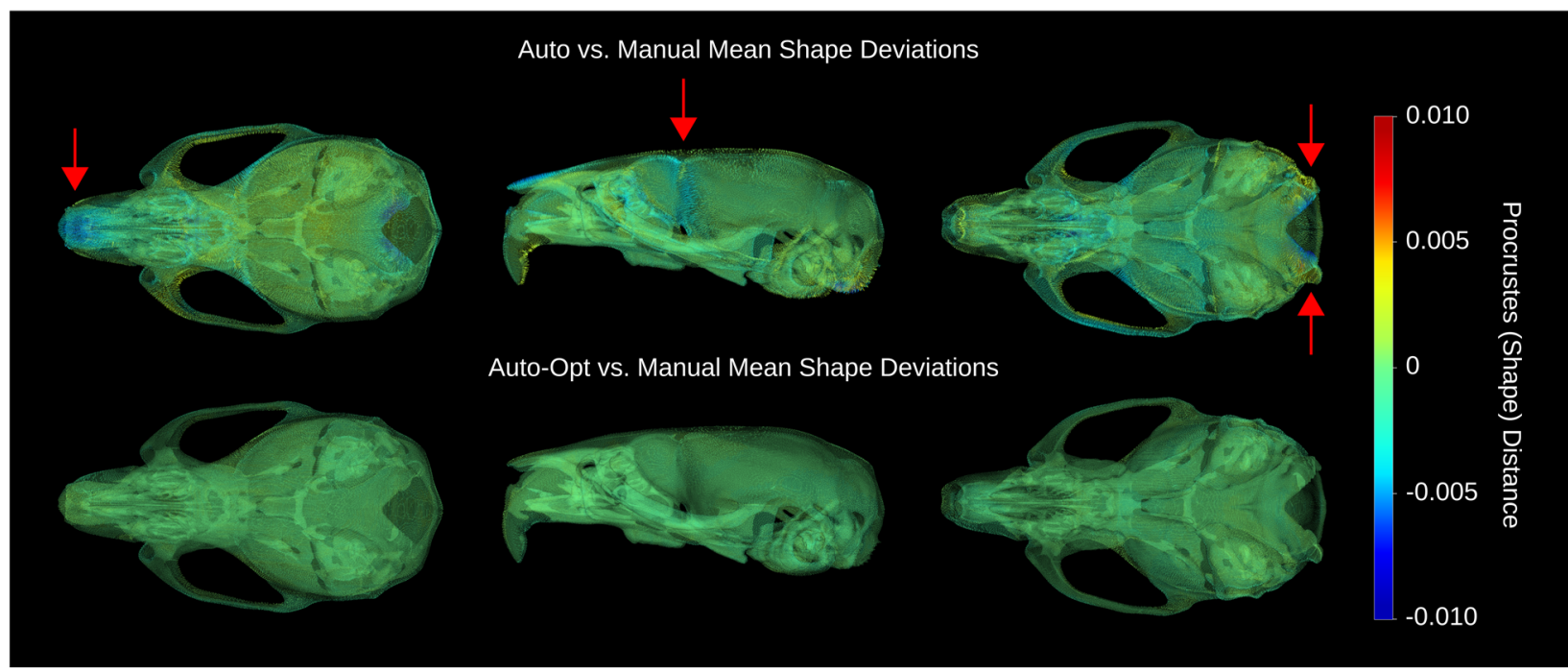

Figure 5. Validation of adult crania test set. (a) Left: Regression of automated-manual Euclidean distances (error) on cross-correlation, a measure of the final target-reference image similarity. Right: Boxplots showing the distribution of cross-correlation values within each developmental stage. (b) Correlation of automated and manual PC scores. Left: Baseline automated PC correlations. Right: Optimized automated

513 PC correlations. (c) Mean shape deviations between the automated and manual datasets. Red arrows indicate error prone areas. 
To analyze mean shape deviations, we computed the grand mean shape for the manual landmarks and deformed it to the automated mean shapes via thin-plate spline. We then used the Morpho package $\mathrm{e}^{101}$ in R to generate a deformation heatmap of Procrustes distances at every vertex of the deformed mesh (Fig.

517 5c). Procrustes distance is equivalent to the root mean squared error between two configurations in shape

518 space. The total distance between the baseline automated mean and manual mean was 0.05 , whereas the

519 distance between the optimized automated mean and manual mean was 0.01 . Visually, the baseline

520 automated mean shape is largely indistinguishable from the manual mean shape, apart from several known

521 problematic areas ${ }^{42}$. First, the anterior extent of the frontonasal prominence is underestimated. Second, the

522 shape of the foramen magnum is altered. Third, the lateral extent of the frontal bone is underestimated,

523 likely because there are no sparse landmarks to interpolate there; however, this area is well-covered by the

524 dense landmark configurations. Optimization successfully corrected errors at these problematic locations.

\section{Outliers and stage-specific shape distributions}

526 For each stage, we calculated the Procrustes distance between the mean shape and every configuration to 527 obtain shape distributions and identify outliers (Fig. S1). We defined outlier shapes as those with a

528 Procrustes distance above $Q_{3}+1.5 \times I Q R$, where $Q_{3}$ is the third quartile and $I Q R$ is the interquartile range.

529 Next, we displayed a minimum threshold isosurface of each outlier image alongside its landmarks to assess

530 the errors. Landmark (.tag) files with clear head registration errors were removed. We observed most errant

531 outlier landmark configurations in the E15.5 and E18.5 embryos, which underwent whole-body

532 registrations. Since the orientation of the head relative to the body cannot be standardized in embryos, the

533 whole-body registrations and inherent constraints of spatial normalization resulted in local registrations

534 errors if their orientation was markedly different from the atlas.

Eliminating problematic outliers with distance distributions is a global solution but not always a

536 local one. For example, if a landmark configuration hardly deviates from the mean on average, yet still has

537 several landmarks with high detection errors, its distance to the mean could be small but its shape distinct. 


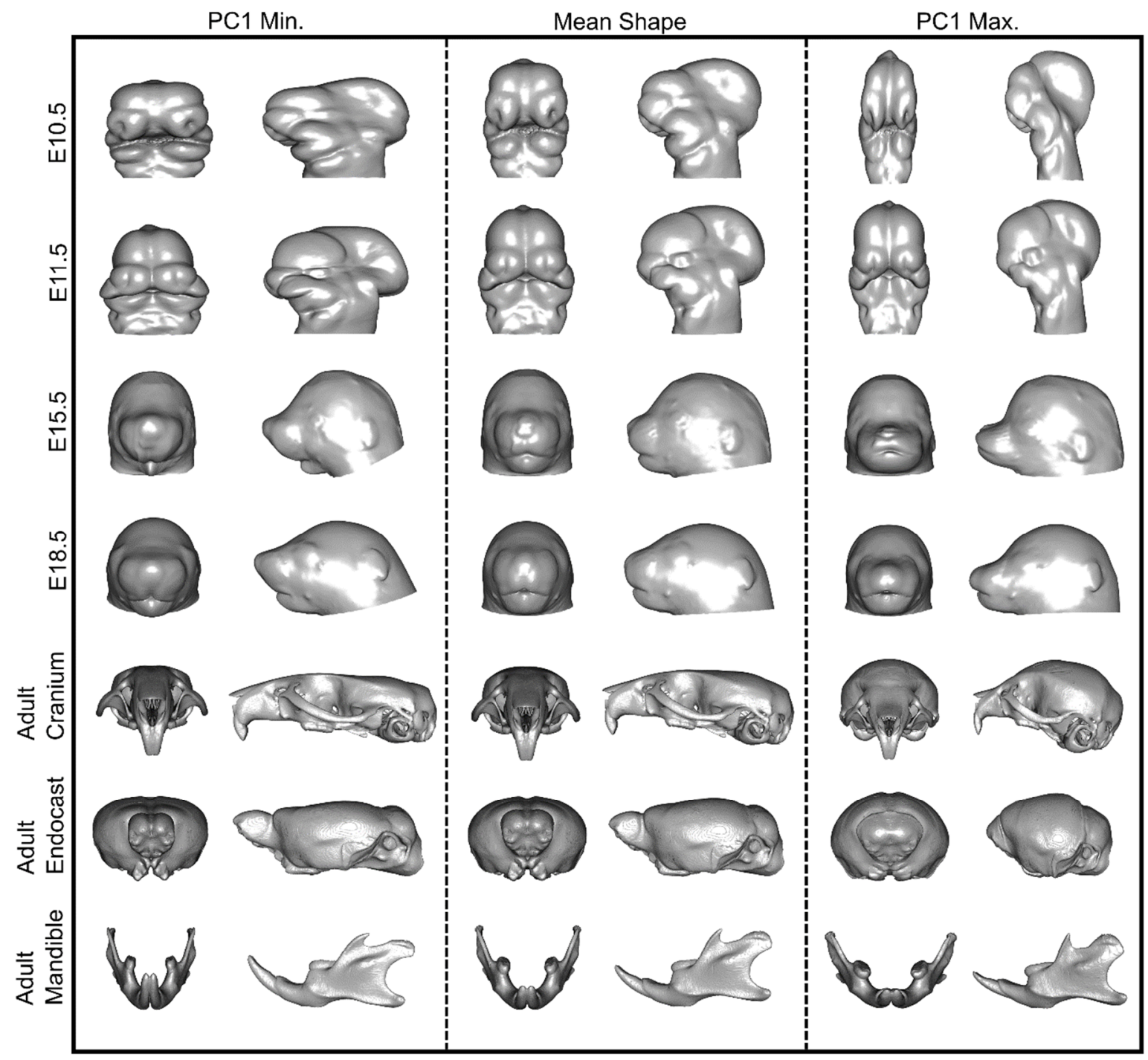

Figure 6. Principal Component Analysis of stage-specific shape data. The mean shape (center) was deformed to the minimum (left) and maximum (right) extremes of PC1. Every morph is shown with anterior and lateral views. Each row represents a different developmental stage, ranging from E10.5 to adulthood.

541 shape to each extreme via thin-plate spline and visualized the outputs. If the deformed surface was unusual,

542 we displayed the image and landmarks as above, removed the errant landmark (.tag) file if necessary, and 543 repeated this process until the prediction was correct. 


\section{Discussion}

\section{Why MusMorph?}

The goal of MusMorph was to create a database of standardized mouse morphology data using an automated, high-throughput, and open-source phenotyping pipeline. By combining developmental atlases with a registration and deep learning framework, we constructed common coordinate systems into which various phenotypic data can be integrated. We primarily focused on acquiring morphological data, including anatomical landmarks, segmentations, and deformation fields, for the craniofacial complex and brain. However, we also generated whole body data for other integrative analyses of late-gestation embryos.

552 To enable novel morphometric analyses of genotype-phenotype maps, we utilized mouse models with 553 substantial developmental and genetic variation. Paired alongside other key metadata, such as strain and 554 sex, MusMorph provides the community with a unique opportunity to disentangle the mechanistic basis for 555 morphological variation.

While sparse landmarks are invaluable for geometric morphometrics, there are scenarios where

557 local shape change can be poorly represented. More ambiguous anatomy, such as curves and surfaces, cannot be sufficiently captured with fixed anatomical landmarks, and semi-landmarking each specimen can be tedious and error-prone. Our standardized sparse and dense landmark datasets can enable global and local shape analyses ${ }^{102,103}$, an area in geometric morphometrics historically overlooked. Homologous dense

561 landmark patches across the embryo datasets will also permit joint superimposition of multiple stages into 562 a common shape space for increased statistical power as well as analyses of ontogeny (Fig. S4). In addition

563 to landmarks, we make the corresponding deformation fields available on an ad hoc basis to support voxel-

564 based meta-analyses of morphology. Despite its ubiquitous application in neuroimaging, voxel-based 565 morphometry is rarely seen in fields that study hard tissue, such as evolutionary developmental biology, 566 anthropology, and paleontology. These deformation fields will let one examine internal and external tissue

567 interactions within anatomical context. Finally, we include anatomical segmentations for several stages, 
which can be used to restrict the dimensionality of a voxel-wise analysis, calculate the size (e.g., volume or surface area) of a structure, or perform a surface-based morphometry analysis. If users are dissatisfied with the coverage of existing landmarks and segmentations, they can modify the atlases and use the image transformations to generate new labels.

We have made the data and scripts freely available at FaceBase (www.facebase.org, doi.org/10.25550/3-HXMC) ${ }^{35}$ and GitHub (https://github.com/jaydevine/MusMorph) to promote transparency, reproducibility, and future data aggregation. Completely open-source efforts like MusMorph are critical for standardizing phenotypic datasets. Unlike the field of genomics, which has been revolutionized through standardized sequencing and data crowdsourcing, phenomics continues to be limited multimodal data integration across the phenomic hierarchy, ranging from cellular and developmental

582 phenotyping with light sheet microscopy ${ }^{104}$ to tissue phenotyping with magnetic resonance imaging and 583 contrast-enhanced computed tomography ${ }^{38}$. Large phenotypic datasets will ultimately give us the statistical 584 power needed to interrogate mechanisms that bias and generate morphological variation. masquerade as anatomy and deceive the registration, even if the alignment is constrained with a mask. We

590 mitigated labelling errors by registering thresholded images and by employing other preprocessing 591 techniques, such as intensity bias correction and normalization. However, in some cases, the intensities of 
the scanning tube could not be distinguished from the specimen, leading to surface landmark errors. Another spatial alignment problem that was difficult to reconcile was variation in articulated anatomical positions. For example, head orientation relative to the body varied widely among the E15.5 and E18.5 datasets, and mandible orientation relative to the skull differed across the adult dataset. We chose to register the entire scan instead of separate segmentations, masks or cropped volumes, because a) a single registration field is computationally more feasible to generate, store, and use downstream and b) a single atlas with a detailed set of labels is better for data standardization. errors in areas of high morphological variability. Lastly, it is important to consider the computational time and memory needed for volumetric registration. To integrate new data, we strongly encourage users to parallelize their work on compute clusters.

\section{Future development}

608 The majority of MusMorph is composed of head data, because we had reservations about registering whole

609 body data. Now that we have observed no significant differences in registration quality among the datasets,

610 we plan to experiment with more whole-body data for embryos and adults. Another area we intend to

611 improve is our developmental coverage. Despite sampling across most of development, we recognize that

612 additional embryo timepoints (e.g., E9.5 and E12.5-14.5) are needed, as are higher sample sizes throughout

613 mid-gestation and early adulthood. The developing mouse craniofacial complex, for example, undergoes

614 immense growth during the first 30 days after birth ${ }^{105}$. Early postnatal datasets will be critical for asking

615 questions about size and ontogenetic allometry. Finally, to complement our large sample of homozygous 
616 embryo mutants, we hope to introduce more wildtype and heterozygous embryos for analyses of normal

617 variation. Heterozygotes have not been a focus of the IMPC, so there is ample opportunity to reveal

618 previously unrecognized embryo phenotypes with standardized MusMorph comparisons. The adult dataset,

619 by contrast, needs to be balanced with more homozygous mutants to better understand how mutations of

620 large effect influence morphological variance and other related phenomena, such as integration and

621 modularity.

\section{Usage Notes}

623 MusMorph is categorized as a "Project" on FaceBase. Projects can be found in the "Data Browser: Projects"

624 tab at the top of the home page. Project data are organized hierarchically. The levels of the hierarchy in 625 ascending order of data specificity are "Project", "Dataset", "Experiment", and "Biosample". A project 626 contains datasets, which are sets of similar studies. Each dataset is annotated with study abstracts, 627 experimental designs, and metadata identifiers. Datasets are composed of experiments. An experiment 628 represents a set of similar specimens, so mice with the same genetic background, age, treatment, and 629 mutation would constitute one experiment. Experiments contain biosamples. A biosample is an individual 630 specimen.

After creating a free account and logging in the MusMorph data and metadata can be downloaded 632 at any level in the project hierarchy using the "Export: BDBag" tool at the top-right of the browser. This 633 export function uses DERIVA ${ }^{106}$, the software platform that powers FaceBase, to generate a BDBag (Big

634 Data Bag) ${ }^{107}$ ZIP file. Users then need to download the file and process it via BDBag client tools, either via 635 the command line or GUI application. Specific details about the DERIVA Client installation and the step636 by-step export instructions are available here: www.facebase.org/help/exporting.

\section{Code Availability}


638 Our code is freely available at https://github.com/jaydevine/MusMorph. The scripts describe every stage of

639 the MusMorph data acquisition and analysis, including image preprocessing (e.g., file conversion, image

640 resampling and intensity correction), processing (e.g., atlas generation, non-linear registration, label

641 propagation), and postprocessing (e.g., shape optimization, morphometric analysis). We developed and

642 implemented the code with Bash 4.4.20, R 3.6.1, Python 3.6, and Julia 1.2.0 on Ubuntu. All code is

643 distributed under the GNU General Public License v3.0.

\section{Acknowledgements}

645 We thank the Advanced Research Computing team at the University of Calgary for facilitating image

646 processing and storage on the ARC and Helix compute clusters. We also thank FaceBase and the

647 International Mouse Phenotyping Consortium for assisting with image data storage and acquisition. Finally,

648 we would like to acknowledge funding from a CIHR Foundation Grant (\#159920), an NSERC Discovery

649 Grant (\#238992-17), an NIH R01 (\#2R01DE019638), an NIH U01 (\#U01DE028729), Alberta Innovates,

650 and the Alberta Children's Hospital Research Institute.

\section{Author contributions}

652 J.D., M.V.G., and B.H.: Study design, image processing, data collection, data analysis, drafting the 653 manuscript, and revising it critically. H.W. and R.S.M.: Study design, data collection, drafting the 654 manuscript, and revising it critically. W.L., A.N., and L.D.L.: Image processing, data collection, and 655 revising the manuscript critically. R.E.S and A.B.: Data upload and organization. R.M.G., H.A.R., M.M., 656 C.M.U., A.C.N., N.M.Y., P.N.G., C.R., C.J.P., T.W., L.N., A.L.C., A.D.L, A.V., F.R.J., J.M.C., O.K., 657 R.Y.B., A.E.M., R.R.A., D.G., W.D., B.R., M.H., and S.A.M.: Data collection and revising the manuscript 658 critically. All authors gave final approval for publication.

\section{Competing interests}

660 The authors declare no competing interests. 


\section{References}

662

663

664

665

666

667

668

669

670

671

672

673

674

675

676

677

678

679

680

681

682

683

684

685

686

687

688

689

690

691

692

693

694

695

696

697

698

699

700

701

702

703

704

1. Hallgrímsson, B., Mio, W., Marcucio, R.S. \& Spritz, R. Let's face it-complex traits are just not that simple. PLoS Genet 10, e1004724, https://doi.org/10.1371/journal.pgen.1004724 (2014).

2. Mitteroecker, P., Cheverud, J. M. \& Pavlicev, M. Multivariate analysis of genotype-phenotype association. Genetics 202, 1345-1363, https://doi.org/10.1534/genetics.115.181339 (2016).

3. Pavlicev, M., Norgard, E. A., Fawcett, G. L. \& Cheverud, J. M. Evolution of pleiotropy: epistatic interaction pattern supports a mechanistic model underlying variation in genotype-phenotype map. J. Exp. Zool. (Mol. Dev. Evol.) 316, 371-385, https://doi.org/10.1002/jez.b.21410 (2011).

4. Green, R. M. et al. Developmental nonlinearity drives phenotypic robustness. Nat. Commun. 8, 1-12, https://doi.org/10.1038/s41467-017-02037-7 (2017).

5. Young, N. M., Chong, H. J., Du, H., Hallgrímsson, B. \& Marcucio, R. S. Quantitative analyses link modulation of sonic hedgehog signaling to continuous variation in facial growth and shape. Development 137, 3405-3409, https://doi.org/10.1242/dev.052340 (2010).

6. Wagner, G. P. Evolution of gene networks by gene duplications: a mathematical model and its implications on genome organization. Proc. Natl. Acad. Sci. U. S. A. 91, 4387-4391, https://doi.org/10.1073/pnas.91.10.4387 (1994).

7. Wagner, G. P. \& Zhang, J. The pleiotropic structure of the genotype-phenotype map: the evolvability of complex organisms. Nat. Rev. Genet. 12, 204-213, https://doi.org/10.1038/nrg2949 (2011).

8. Rice, S. H. The evolution of canalization and the breaking of von Baer's laws: modeling the evolution of development with epistasis. Evolution 52, 647—656, https://doi.org/10.1111/j.15585646.1998.tb03690.x (1998).

9. Rice, S. H. Theoretical approaches to the evolution of development and genetic architecture. Ann. N.Y. Acad. Sci. 1133, 67-86, https://doi.org/10.1196/annals.1438.002 (2008).

10. Hallgrímsson, B. et al. The developmental-genetics of canalization. In Seminars in Cell \& Developmental Biology, Vol. 88 (Academic Press, 2019). https://doi.org/10.1016/j.semcdb.2018.05.019.

11. Karim, K. et al. Xenbase: a genomic, epigenomic and transcriptomic model organism database. Nucleic Acids Res. 46, D861—D868, https://doi.org/10.1093/nar/gkx936 (2018).

12. Blake, J. A. et al. The Mouse Genome Database (MGD): the model organism database for the laboratory mouse. Nucleic Acids Res. 30, 113-115, https://doi.org/10.1093/nar/30.1.113 (2002).

13. Howe et al. ZFIN, the Zebrafish Model Organism Database: increased support for mutants and transgenics. Nucleic Acids Res. 41, D854-D860, https://doi.org/10.1093/nar/gks938 (2012).

14. Wang, L., Wang, S., Li, Y., Paradesi, M. S. R. \& Brown, S. J. BeetleBase: the model organism database for Tribolium castaneum. Nucleic Acids Res. 35, D476-D479, https://doi.org/10.1093/nar/gk1776 (2006).

15. Brown, S. D. \& Moore, M. W. The International Mouse Phenotyping Consortium: past and future perspectives on mouse phenotyping. Mamm. Genome 23, 632-640, https://doi.org/10.1007/s00335-012-9427-x (2012).

16. Brown, S. D. \& Moore, M. W. Towards an encyclopaedia of mammalian gene function: The International Mouse Phenotyping Consortium. Dis. Model. Mech. 5, 289-292, https://doi.org/10.1242/dmm.009878 (2012).

17. Horner, N. R. et al. LAMA: automated image analysis for the developmental phenotyping of mouse embryos. Development 148, dev192955, https://doi.org/10.1242/dev.192955 (2021). 
18. Koscielny, G. et al. The International Mouse Phenotyping Consortium Web Portal, a unified point of access for knockout mice and related phenotyping data. Nucleic Acids Res. 42, D802D809, https://doi.org/10.1093/nar/gkt977 (2014).

19. Meehan, T. F. et al. Disease model discovery from 3,328 gene knockouts by The International Mouse Phenotyping Consortium. Nat. Genet. 49, 1231-1238, https://doi.org/10.1038/ng.3901 (2017).

20. Dickinson, M. E. et al. High-throughput discovery of novel developmental phenotypes. Nature 537, 508-514, https://doi.org/10.1038/nature19356 (2016).

21. Churchill, G. A., Gatti, D. M., Munger, S. C. \& Svenson, K. L. The diversity outbred mouse population. Mamm. Genome 23, 713-718, https://doi.org/10.1007/s00335-012-9414-2 (2012).

22. Collaborative Cross Consortium. The genome architecture of the Collaborative Cross mouse genetic reference population. Genetics 190, 389-401, https://doi.org/10.1534/genetics.111.132639 (2012).

23. Katz, D. C. et al. Facial shape and allometry quantitative trait locus intervals in the Diversity Outbred mouse are enriched for known skeletal and facial development genes. PLoS ONE 15, e023337, https://doi.org/10.1371/journal.pone.0233377 (2020).

24. Ashburner, J. \& Friston, K. J. Voxel-based morphometry-the methods. NeuroImage 11, 805821, https://doi.org/10.1006/nimg.2000.0582 (2000).

25. Fonov, V. et al. Unbiased average age-appropriate atlases for pediatric studies. NeuroImage 54, 313-327, https://doi.org/10.1016/j.neuroimage.2010.07.033 (2011).

26. Ridgway, G. R. et al. Ten simple rules for reporting voxel-based morphometry studies. NeuroImage 40, 1429-1435, https://doi.org/10.1016/j.neuroimage.2008.01.003 (2008).

27. Silver, M., Montana, G., Nichols, T. E. \& Alzheimer's Disease Neuroimaging Initiative. False positives in neuroimaging genetics using voxel-based morphometry data. NeuroImage 54, 9921000, https://doi.org/10.1016/j.neuroimage.2010.08.049 (2011).

28. Adams, D. C., Rohlf, F. J. \& Slice, D. E. A field comes of age: geometric morphometrics in the 21st century. Hystrix 24, 7, https://doi.org/10.4404/hystrix-24.1-6283 (2013).

29. Boyer, D. M. et al. A new fully automated approach for aligning and comparing shapes. Anat. Rec. 298, 249-276, https://doi.org/10.1002/ar.23084 (2015).

30. Maga, A. M., Tustison, N. J. \& Avants, B. B. A population level atlas of Mus musculus craniofacial skeleton and automated image-based shape analysis. J. Anat. 231, 433-443, https://doi.org/10.1111/joa.12645 (2017).

31. Porto, A. \& Voje, K. L. ML-morph: A fast, accurate and general approach for automated detection and landmarking of biological structures in images. Methods Ecol. Evol. 11, 500_512, https://doi.org/10.1111/2041-210X.13373 (2020).

32. Rolfe, S. et al. SlicerMorph: An open and extensible platform to retrieve, visualize and analyze 3D morphology. bioRxiv, https://doi.org/10.1101/2020.11.09.374926 (2020).

33. Vidal-García, M., Bandara, L. \& Keogh, J. S. ShapeRotator: an R tool for standardized rigid rotations of articulated three-dimensional structures with application for geometric morphometrics. Ecol. Evol. 8, 4669-4675, https://doi.org/10.1002/ece3.4018 (2018).

34. Samuels, B. D. et al. FaceBase 3: analytical tools and FAIR resources for craniofacial and dental research. Development 147, dev191213, https://doi.org/10.1242/dev.191213 (2020).

35. Devine, J. et al. MusMorph, a database of standardized mouse morphology data for morphometric meta-analyses. FaceBase Consortium, https://doi.org/10.25550/3-HXMC (2021).

36. Devine, J. et al. Project-wide metadata, atlases, and landmarks for MusMorph. FaceBase Consortium, https://doi.org/10.25550/6-2EPY (2021). 
37. Wong, M. D., Spring, S. \& Henkelman, R. M. Structural stabilization of tissue for embryo phenotyping using micro-CT with iodine staining. PLOS ONE 8, e84321, https://doi.org/10.1371/journal.pone.0084321 (2013).

38. Gignac, P. M. et al. Diffusible iodine-based contrast-enhanced computed tomography (diceCT): an emerging tool for rapid, high-resolution, 3-D imaging of metazoan soft tissues. J. Anat. 228, 889-909, https://doi.org/10.1111/joa.12449 (2016).

39. Green, R. M., Leach, C. L., Hoehn, N., Marcucio, R. S. \& Hallgrímsson, B. Quantifying threedimensional morphology and RNA from individual embryos. Dev. Dynam. 246, 431-436, https://doi.org/10.1002/DVDY.24490 (2017).

40. Feldkamp, L. A., Davis, L. C. \& Kress, J. W. Practical cone-beam algorithm. J. Opt. Soc. Am. A 1, 612-619, https://doi.org/10.1364/JOSAA.1.000612 (1984).

41. Vincent, R. D. et al. MINC 2.0: a flexible format for multi-modal images. Front. Neuroinform. 10, 35, https://doi.org/10.3389/fninf.2016.00035 (2016).

42. Sled, J. G., Zijdenbos, A. P. \& Evans, A. C. A nonparametric method for automatic correction of intensity nonuniformity in MRI data. IEEE T. Med. Imaging 17, 87-97, https://doi.org/10.1109/42.668698 (1998).

43. Friedel, M., van Eede, M. C., Pipitone, J., Chakravarty, M. M. \& Lerch, J. P. Pydpiper: a flexible toolkit for constructing novel registration pipelines. Front. Neuroinform. 8, 67, https://doi.org/10.3389/fninf.2014.00067 (2014).

44. Percival, C. J. et al. The effect of automated landmark identification on morphometric analyses. $J$. Anat. 234, 917-935, https://doi.org/10.1111/joa.12973 (2019).

45. Collins, D. L., Neelin, P., Peters, T. M. \& Evans, A. C. Automatic 3D intersubject registration of MR volumetric data in standardized Talairach space. J. Comput. Assist. Tomo. 18, 192-205, https://doi.org/10.1097/00004728-199403000-00005 (1994).

46. Lerch, J. P., Sled, J. G. \& Henkelman, R. M. MRI Phenotyping of Genetically Altered Mice. In: Magnetic Resonance Neuroimaging. Methods in Molecular Biology (Methods and Protocols), Vol. 711 (eds. Modo M., Bulte, J.) (Humana Press, 2011). https://doi.org/10.1007/978-1-61737992-5_17.

47. Collins, D. L. \& Evans, A. C. Animal: validation and applications of nonlinear registration-based segmentation. Int. J. Pattern Recogn. 11, 1271-1294, https://doi.org/10.1142/S0218001497000597 (1997).

48. Wong, M. D., Dorr, A. E., Walls, J. R., Lerch, J. P. \& Henkelman, R. M. A novel 3D mouse embryo atlas based on micro-CT. Development 139, 3248-3256, https://doi.org/10.1242/dev.082016 (2012).

49. Kikinis R., Pieper S. D. \& Vosburgh K. G. 3D Slicer: A Platform for Subject-Specific Image Analysis, Visualization, and Clinical Support. In: Intraoperative Imaging and Image-Guided Therapy (eds. Jolesz, F.) (Springer, 2014). https://doi.org/10.1007/978-1-4614-7657-3_19.

50. Percival, C. J., Green, R., Marcucio, R. S. \& Hallgrímsson, B. Surface landmark quantification of embryonic mouse craniofacial morphogenesis. BMC Dev. Biol. 14, 1-12, https://doi.org/10.1186/1471-213X-14-31 (2014).

51. Bastir, M. A systems-model for the morphological analysis of integration and modularity in human craniofacial evolution. J. Anthropol. Sci. 86, 19934468 (2008).

52. Porto, A., de Oliveira F. B., Shirai, L. T., De Conto, V. \& Marroig, G. The evolution of modularity in the mammalian skull I: Morphological integration patterns and magnitudes. Evol. Biol. 36, 118-135, https://doi.org/10.1007/s11692-008-9038-3 (2009).

53. Hallgrímsson, B. et al. Integration and the developmental genetics of allometry. Integr. Comp. Biol. 59, 1369-1381, https://doi.org/10.1093/icb/icz105 (2019). 
54. Richtsmeier, J. T. et al. Phenotypic integration of neurocranium and brain. J. Exp. Zool. (Mol. Dev. Evol.) 306, 360-378, https://doi.org/10.1002/jez.b.21092 (2006).

55. Marchini, M. et al. Wnt signaling drives correlated changes in facial morphology and brain shape. Front. Cell Dev. Biol. 9, 694, https://doi.org/10.3389/fcell.2021.644099 (2021).

56. Smith, K. K. Integration of craniofacial structures during development in mammals. Am. Zool. 36, 70-79 (1996).

57. Young, N. M., Linde-Medina, M., Fondon, J. W., Hallgrímsson, B. \& Marcucio, R. S. Craniofacial diversification in the domestic pigeon and the evolution of the avian skull. Nat. Ecol. Evol. 1, 1-8, https://doi.org/10.1038/s41559-017-0095 (2017).

58. Toussaint, N. et al. A landmark-free morphometrics pipeline for high-resolution phenotyping: application to a mouse model of Down syndrome. Development 148, dev188631, https://doi.org/10.1242/dev.188631 (2021).

59. Avants, B. B. et al. A reproducible evaluation of ANTs similarity metric performance in brain image registration. NeuroImage 54, 2033-2044, https://doi.org/10.1016/j.neuroimage.2010.09.025 (2011).

60. Devine, J. et al. A registration and deep learning approach to automated landmark detection for geometric morphometrics. Evol. Biol. 47, 246-259, https://doi.org/10.1007/s11692-020-09508-8 (2020).

61. Attanasio, C. et al. Fine tuning of craniofacial morphology by distant-acting enhancers. Science 342, 1-20, https://doi.org/10.1126/science.1241006 (2014).

62. Hallgrímsson, B., Willmore, K., Dorval, C. \& Cooper, D. M. L. Craniofacial variability and modularity in macaques and mice. J. Exp. Zool. Part B 302, 207-225, https://doi.org/10.1002/jez.b.21002 (2004).

63. Hallgrímsson, B. et al. The Brachymorph mouse and the developmental-genetic basis for canalization and morphological integration. Evol. Dev. 8, 61-73, https://doi.org/10.1111/j.1525142X.2006.05075.x (2006).

64. Hallgrímsson, B. et al. Deciphering the Palimpsest: Studying the relationship between morphological integration and phenotypic covariation. Evol. Biol. 36, 355-376, https://doi.org/10.1007/s11692-009-9076-5 (2009).

65. Lieberman, D. E., Hallgrímsson, B., Liu, W., Parsons, T. E. \& Jamniczky, H. A. Spatial packing, cranial base angulation, and craniofacial shape variation in the mammalian skull: Testing a new model using mice. J. Anat. 212, 720—735, https://doi.org/10.1111/j.1469-7580.2008.00900.x (2008).

66. Devine, J. et al. Collaborative Cross: A standardized mouse morphology dataset for MusMorph. FaceBase Consortium, https://doi.org/10.25550/3-KB0W (2021).

67. Devine, J. et al. Hybrid: A standardized mouse morphology dataset for MusMorph. FaceBase Consortium, https://doi.org/10.25550/3-KB32 (2021).

68. Devine, J. et al. Diversity Outbred: A standardized mouse morphology dataset for MusMorph. FaceBase Consortium, https://doi.org/10.25550/3-KB0W (2021).

69. Devine, J. et al. IMPC: A standardized mouse morphology dataset for MusMorph. FaceBase Consortium, https://doi.org/10.25550/3-JZA6 (2021).

70. Devine, J. et al. Ap2: A standardized mouse morphology dataset for MusMorph. FaceBase Consortium, https://doi.org/10.25550/3-JQMG (2021).

71. Devine, J. et al. B9d: A standardized mouse morphology dataset for MusMorph. FaceBase Consortium, https://doi.org/10.25550/3-JQMM (2021).

72. Devine, J. et al. Bulgy: A standardized mouse morphology dataset for MusMorph. FaceBase Consortium, https://doi.org/10.25550/3-JZ9G (2021). 
73. Devine, J. et al. Brain-Face: A standardized mouse morphology dataset for MusMorph. FaceBase Consortium, https://doi.org/10.25550/3-KB3J (2021).

74. Devine, J. et al. Longshanks: A standardized mouse morphology dataset for MusMorph. FaceBase Consortium, https://doi.org/10.25550/3-KFBE (2021).

75. Unger, C. M., Devine, J., Hallgrímsson, B. \& Rolian, C. Selection for increased tibia length in mice alters skull shape through parallel changes in developmental mechanisms. Elife 10, e67612, https://doi.org/10.7554/eLife.67612 (2021).

76. Marchini, M. \& Rolian, C. Artificial selection sheds light on developmental mechanisms of limb elongation. Evolution 72, 825-837, https://doi.org/10.1111/evo.13447 (2018).

77. Spring, S., Lerch, J. P. \& Henkelman, R. M. Sexual dimorphism revealed in the structure of the mouse brain using three-dimensional magnetic resonance imaging. NeuroImage 35, 1424-1433, https://doi.org/10.1016/j.neuroimage.2007.02.023 (2007).

78. Gonzalez, P. N., Bernal, V. \& Perez, S. I. Analysis of sexual dimorphism of craniofacial traits using geometric morphometric techniques. Int. J. Osteoarchaeol. 21, 82-91, https://doi.org/10.1002/oa.1109 (2011).

79. Callewaert, F. et al. Sexual dimorphism in cortical bone size and strength but not density is determined by independent and time-specific actions of sex steroids and IGF-1: Evidence from pubertal mouse models. J. Bone Miner. Res. 25, 617-626, https://doi.org/10.1359/jbmr.090828 (2010).

80. Grove, K. L., Fried, S. K., Greenberg, A. S., Xiao, X. Q. \& Clegg, D. J. A microarray analysis of sexual dimorphism of adipose tissues in high-fat-diet-induced obese mice. Int. J. Obesity 34, 989-1000, https://doi.org/10.1038/ijo.2010.12 (2010).

81. Eaton, M. et al. Complex patterns of cell growth in the placenta in normal pregnancy and as adaptations to maternal diet restriction. PLOS ONE 15, e 0226735 , https://doi.org/10.1371/journal.pone.0226735 (2020).

82. Gonzalez et al. Chronic protein restriction in mice impacts placental function and maternal body weight before fetal growth. PLoS ONE 11, e0152227, https://doi.org/10.1371/journal.pone.0152227 (2016).

83. Devine, J. et al. Strain Comparison: A standardized mouse morphology dataset for MusMorph. FaceBase Consortium, https://doi.org/10.25550/3-JZ9J (2021).

84. Jamniczky, H. A. \& Hallgrímsson, B. A comparison of covariance structure in wild and laboratory muroid crania. Evolution 63, 1540—1556, https://doi.org/10.1111/j.15585646.2009.00651.x (2009).

85. Davies, A. G., Bettinger, J. C., Thiele, T. R., Judy, M. E. \& McIntire, S. L. Natural variation in the npr-1 gene modifies ethanol responses of wild strains of C. elegans. Neuron 42, 731-743, https://doi.org/10.1016/j.neuron.2004.05.004 (2004).

86. Pavlicev, M., Norgard, E. A., Fawcett, G. L. \& Cheverud, J. M. Evolution of pleiotropy: epistatic interaction pattern supports a mechanistic model underlying variation in genotype-phenotype map. J. Exp. Zool. (Mol. Dev. Evol.) 316, 371-385 (2011).

87. Percival, C. J., Marangoni, P., Tapaltsyan, V., Klein, O. \& Hallgrímsson, B. The interaction of genetic background and mutational effects in regulation of mouse craniofacial shape. G3-Genes Genom. Genet. 7, 1439-1450, https://doi.org/10.1534/g3.117.040659 (2017).

88. Devine, J. et al. Spry: A standardized mouse morphology dataset for MusMorph. FaceBase Consortium, https://doi.org/10.25550/3-JZAM (2021).

89. Cheverud, J. M. Relationships among ontogenetic, static, and evolutionary allometry. Am. J. Phys. Anthropol. 59, 139-149 (1982). 
90. Gonzalez, P. N., Kristensen, E., Morck, D. W., Boyd, S. \& Hallgrímsson, B. Effects of growth hormone on the ontogenetic allometry of craniofacial bones. Evol. Dev. 15, 133-145, https://doi.org/10.1111/ede.12025 (2013).

91. Klingenberg, C. P. Multivariate allometry. In Advances in Morphometrics (Springer, 1996). https://doi.org/10.1007/978-1-4757-9083-2_3.

92. Mosimann, J. E. Size allometry: size and shape variables with characterizations of the lognormal and generalized gamma distributions. J. Am. Stat. Assoc. 65, 930-945, https://doi.org/10.1080/01621459.1970.10481136 (1970).

93. Jolicoeur, P. Note: the multivariate generalization of the allometry equation. Biometrics 19, 497-499, https://doi.org/10.2307/2527939 (1963).

94. Richtsmeier, J. T. \& Flaherty, K. Hand in glove: brain and skull in development and dysmorphogenesis. Acta. Neuropathol. 125, 469-489 (2013).

95. Klingenberg, C. P. Morphometrics and the role of the phenotype in studies of the evolution of developmental mechanisms. Gene 287, 3-10, https://doi.org/10.1016/S0378-1119(01)00867-8 (2002).

96. Soulé, M. E. Heterozygosity and developmental stability: another look. Evolution 33, 396-401, https://doi.org/10.2307/2407629 (1979).

97. Sotiras, A., Davatzikos, C. \& Paragios, N. Deformable medical image registration: A survey. IEEE T. Med. Imaging 32, 1153-1190, https://doi.org/10.1109/TMI.2013.2265603 (2013).

98. Devine, J. et al. Ghrhr: A standardized mouse morphology dataset for MusMorph. FaceBase Consortium, https://doi.org/10.25550/3-KB08 (2021).

99. Vidal-García, M. morpho. Tools. GM v1.0.0: A set of R tools to help with geometric morphometric analyses of 3D data. https://doi.org/10.5281/zenodo.4673771 (2021).

100. Wong, M. D. et al. 4D atlas of the mouse embryo for precise morphological staging. Development 142, 3583-3591, https://doi.org/10.1242/dev.125872 (2015).

101. Schlager, S. Morpho and Rvcg-Shape analysis in R: R packages for geometric morphometrics, shape analysis and surface manipulations. In Statistical Shape and Deformation Analysis. Methods, Implementation and Applications (Academic Press, 2017). https://doi.org/10.1016/B978-0-12-810493-4.00011-0.

102. Claes, P. et al. Genome-wide mapping of global-to-local genetic effects on human facial shape. Nat. Genet. 50, 414-423, https://doi.org/10.1038/s41588-018-0057-4 (2018).

103. Mitteroecker, P. et al. Morphometric variation at different spatial scales: coordination and compensation in the emergence of organismal form. Syst. Biol. 69, 913-926, https://doi.org/10.1093/sysbio/syaa007 (2020).

104. Epp, J. R. et al. Optimization of CLARITY for clearing whole-brain and other intact organs. eNeuro 2, https://doi.org/10.1523/ENEURO.0022-15.2015 (2015).

105. Vora, S. R., Camci E. D. \& Cox, T. C. Postnatal ontogeny of the cranial base and craniofacial skeleton in male C57BL/6J mice: A reference standard for quantitative analysis. Front. Physiol. 6, 417, https://doi.org/10.3389/fphys.2015.00417 (2016).

106. Bugacov, A. et al. Experiences with DERIVA: An asset management platform for accelerating eScience. In: IEEE $13^{\text {th }}$ International Conference on e-Science, 79-88, https://doi.org/10.1109/eScience.2017.20 (2017).

107. Chard, K. et al. I'll take that to go: Big data bags and minimal identifiers for exchange of large, complex datasets. In: IEEE International Conference on Big Data, 319-328, https://doi.org/10.1109/BigData.2016.7840618 (2016). 